\title{
Application of transverse functions to control differentially driven wheeled robots using velocity fields
}

\author{
D. PAZDERSKI* \\ Chair of Control and Systems Engineering, Poznań University of Technology, 3A Piotrowo St., 60-965 Poznań, Poland
}

\begin{abstract}
This paper deals with control of a nonholonomic unicycle-like robot in a cluttered environment with static obstacles. The proposed solution is based on a combination of a universal motion controller taking advantage of transverse functions with a navigation velocity field determining a path in a free task space. The motion controller is used to imitate an omnidirectional planar kinematics such that nonholonomic constraints become hidden for a navigation layer. Then it is possible to generate vector fields which govern motion of the omnidirectional frame. The controller using the transverse function is discussed in depth. In particular, a possible parametrization of this function is considered and analysis of an augmented dynamics is provided for different motion patterns. Next, construction of obstacles and potential design for star-like shapes are presented. The navigation algorithm is verified experimentally and the results are discussed.
\end{abstract}

Key words: transverse functions, nonholonomic systems, systems on Lie groups, potential functions, velocity fields.

\section{Introduction}

Control of nonholonomic mechanical systems becomes an important issue in robotics since many robots, in particular mobile devices, are subject to nonintegrable kinematic constraints. These constraints impose restrictions on feasible directions in the phase space that lead to significant difficulties in design of a stabilizing state feedback discussed by Brockett [1]. As a result, an application of classic control methods is restricted to the case when the linear approximation of an open-loop dynamics is controllable. This condition can be satisfied when tracking some class of reference trajectories introducing persistence excitation (at least non vanishing reference motion is required) is considered. Following this idea, many feedback controllers dedicated to nonholonomic systems were proposed, [2-5]. In order to cover a more complicated case when linear controllability is lost one needs to use nonlinear techniques based on discontinuous or time-varying feedbacks, cf. [6-9]. However, both approaches have significant drawbacks that hinder achieving asymptotic stabilization at a point with an acceptable performance and robustness to unmodelled dynamics. Additionally, from work done by Lizárraga [10] it follows that no asymptotic smooth stabilizer exists for all feasible trajectories.

In order to overcome theoretical and practical drawbacks concerning stabilization of nonholonomic systems a theory of transverse functions has been formulated by Morin and Samson [11]. So far this method has been applied to various nonhoholonomic systems including simple wheeled robots [12], wheeled robots with trailers [13, 14], nonholonomic ball [15], trident-snake robot and others [16, 17], a three link mechanical structure in the flight phase [18], and manipulators with non-

*e-mail: dariusz.pazderski@put.poznan.pl holonomic constraints $[19,20]$. The key property of the controller using transverse functions is the ability to approximate non feasible directions in the phase space using periodic inputs with tunable frequency. As a result, it is possible to change configuration of a small-time controllable nonholonomic system in almost arbitrarily way (similarly as for a holonomic system). Consequently, it becomes possible to stabilize its configuration in some vicinity of a desired point or trajectory, even when a permanent disturbance occurs. Simultaneously, smoothness of the control law offers the possibility to increase robustness of the closed-loop system to some class of disturbances (the robustness issue concerning control of nonholonomic systems has been reported in some papers, [21-23]).

Another fundamental problem in mobile robotics with a significant practical impact is control in the presence of constraints imposed on a workspace, $[24,25]$. This is the main issue that has to be considered in order to solve the navigation problem in a cluttered environment. So far many approaches based on analytical, combinatorial and probabilistic methods have been proposed to cover this task. The formal analytical methods are usually derived from the concept of potential functions introduced in robotics by Khatib, [26]. A formal analysis of this method was carried out by Rimon and Koditschek, who introduced the so-called navigation function ensuring one global minimum for star obstacles, [27, 28]. The potential function paradigm has proved to be an effective tool for control design also for phase-constrained systems, [29, 30]. A complementary method of motion planning based on potential functions may take advantage of harmonic functions which solve the Laplace equation, [31-34]. The fundamental advantage of these functions is the lack of local minima, $[35,36]$.

Some authors employ non-gradient vector fields in order to find feasible paths for nonholonomic vehicles, [37]. The concept of potential and non gradient fields has been successfully used for multi agent systems, [38, 39]. Recently, an algorithm 
when both kinematic and dynamic constraints are taken into account has been proposed in [40].

In this paper we deal with the unicycle-like robot which is the basic example of nonholonomic systems used in practice. So far robots equipped with two-wheeled differentially driven structure constitutes the most important class of robotic vehicles. This is due to simplicity of this drive as well as relatively high mobility in comparison to a car-like structure.

Here we consider application of the decoupling controller based on a transverse function in order to imitate the omnidirectional planar robot. We borrowed the concept already discussed in [41] and extended it in order to solve another control task. Namely, instead of employing tracking of a reference trajectory directly, it is assumed that an input to the motion controller specifies velocity of the omnidirectional frame. Basically, the algorithm using the transverse function is treated as a bridge connecting nonhonolonomic kinematics with a higher level navigation layer defining directions of motion based on the environment topology. According to the best author's knowledge the considered application based on transverse functions has not been proposed in the literature so far.

The paper is divided into three parts. In the first part, in Section 2, a fundamental mathematical background, notation on a Lie group, a general idea of transverse functions and decoupling controller are recalled. The next part presented in Sections 3 and 4 is focused on detailed analysis of the motion controller designed for the unicycle. In particular the parametrization of the transverse function and stability of an augmented dynamics governing evolution of the transverse function for the given types of motion are discussed in depth. The last part of the paper is focused on the application of the considered control method to solve a navigation task using velocity field. In order to do this potential functions for star-like obstacles and corresponding velocity fields are designed. The algorithm is verified experimentally in Section 6 for various scenarios using a real control system equipped with a laboratory twowheeled robot.

Acknowledgements. This work was supported by the University Grant No. 09/93/DSPB/0611.

\section{Decoupling of nonholonomic systems on Lie group using transverse functions}

2.1. Preliminary and notation. Let $G$ be a Lie group with group operation $g h \in G$, where $g, h \in G$. The inverse element of $g$, denoted by $g^{-1} \in G$, satisfies $g g^{-1}=g^{-1} g=e$, with $e$ being neutral (identity) element of group $G$. The fundamental group diffeomorphisms include: left translation $l_{g}: G \rightarrow G, h \rightarrow g h$, right translation $r_{g}: G \rightarrow G, h \rightarrow h g$ and conjugation $\phi_{g}: G \rightarrow G, h \rightarrow l_{g}\left(r_{g^{-1}}(h)\right)=r_{g^{-1}}\left(l_{g}(h)\right)=g h g^{-1}$. Differentials of these maps are defined by: $d l_{g}(h):=\frac{d}{d h} l_{g}(h)$, $d r_{g}(h):=\frac{d}{d h} r_{g}(h)$ and $d \phi_{g}(h):=\frac{d}{d h} g h g^{-1}$, respectively.

For the given Lie group $G$ associated Lie algebra $\mathfrak{g}$ can be defined. It consists of vector fields $V_{1}, V_{2}, \ldots, V_{i}, \ldots$ on $G$ which are left-invariant under left translation, namely

$$
\forall g, h \in G, d l_{g}(h) V_{i}(h)=V_{i}(g h)
$$

Assume that a basis of $\mathfrak{g}$ is constituted by $n$ independent vector fields $X_{1}, X_{2}, \ldots, X_{n} \in \mathfrak{g}$, where $n=\operatorname{dim} G$. Applying vector-matrix notation this basis can be defined by $X:=\left[X_{1} X_{2} \ldots X_{n}\right] \in \mathbb{R}^{n \times n}$. Accordingly, one can express any vector field $V \in \mathfrak{g}$ evaluated at $g \in G$ in the Lie algebra basis as: $V=\sum_{i=1}^{n} X_{i}(g) w_{i}=X(g) w$, where $w=\left[w_{1} \ldots w_{n}\right]^{\top} \in \mathbb{R}^{n}$.

The Lie algebra $\mathfrak{g}$ of the group $G$ is related with this group via exponential map: $\mathfrak{g} \rightarrow G$, denoted by

$$
g=\exp (X(\cdot) w)
$$

where $g \in G$ and $X(\cdot) w \in \mathfrak{g}$. This map is understood as the solution of differential equation $\frac{d}{d \tau} g=X(g) w$ with $g(0)=e$ evaluated at $\tau=1$.

The other important differential operator is the adjoint operator $A d: G \times \mathfrak{g} \rightarrow \mathfrak{g}$ which is given by $A d(g) V:=d \phi_{g}(e) V$, where $V \in \mathfrak{g}$. For computation purposes we also consider conjugation of $A d$ given by: $A d^{X}(g)=X(e)^{-1} A d(g) X(e)$.

2.2. Kinematic control system on Lie group. Consider $m$ input small-time locally controllable (STLC) system defined on $G$

$$
\dot{g}=\sum_{i=1}^{m} X_{i}(g) u_{i}
$$

where $g \in G$ denotes configuration and $X_{1}, X_{2}, \ldots, X_{m} \in \mathfrak{g}$ are left-invariant control vector fields and $\mathrm{u}_{1}, \mathrm{u}_{2}, \ldots, u_{m}$ denote inputs. Since the given system is STLC one can define the following basis of $\mathfrak{g}$

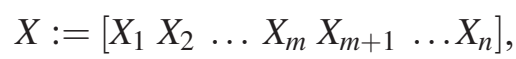

where $X_{m+1}, \ldots, X_{n}$ are properly chosen (first and higher order) Lie brackets of control vector fields $X_{1}, X_{2}, \ldots, X_{m}$. Equivalently, using (4) one can rewrite (3) as follows (cf. [12])

$$
\dot{g}=X(g) C u
$$

where $C:=\left[\begin{array}{ll}I & 0\end{array}\right]^{\top} \in \mathbb{R}^{n \times m}$, with $I \in \mathbb{R}^{m \times m}$ being identity matrix, and $u=\left[\begin{array}{llll}u_{1} & u_{2} & \ldots & u_{m}\end{array}\right]^{\top} \in \mathbb{R}^{m}$.

2.3. Transverse function. Next, we recall a definition of a transverse function defined on a torus.

Definition 1. Let $f: \mathbb{T}^{p} \rightarrow G$, where $p \geq n-m$ be a smooth function defined on $p$ dimensional torus satisfying

$$
\forall \alpha \in \mathbb{T}^{p}, \operatorname{rank} M(\alpha)=n,
$$

while $M(\alpha) \in \mathbb{R}^{n \times(m+p)}$ is defined as follows

$$
M(\alpha):=\left[X_{1}(f(\alpha)) X_{2}(f(\alpha)) \ldots X_{m}(f(\alpha))-\frac{\partial f(\alpha)}{\partial \alpha}\right]
$$

and

$$
\forall \alpha \in \mathbb{T}^{p}, f(\alpha) \in B_{\varepsilon}(e)
$$


where $B_{\varepsilon}(e)$ denotes $n$-dimensional ball with radius $\varepsilon$ and centre $e$. This function is transversal to control vector fields $X_{1}, \ldots, X_{m}$ and is called transverse function (TF) for system (3).

Notice that when $p=n-m$ the transversality condition given by (6) can be written in a more convenient way using basis $X$. Correspondingly, it can be assumed that

$$
\frac{\partial f(\alpha)}{\partial \alpha}:=X(f(\alpha)) A(\alpha)
$$

where $A(\alpha):=\left[A_{1}^{\top} A_{2}^{\top}\right]^{\top} \in \mathbb{R}^{n \times(n-m)}$ is the matrix composed of columns being derivative of $f$ expressed in $X$, while $A_{1} \in \mathbb{R}^{m \times(n-m)}$ and $A_{2} \in \mathbb{R}^{(n-m) \times(n-m)}$. Using (9) and recalling definition of $C$ allows one to rewrite matrix $M$ as follows

$$
M(\alpha)=X(f(\alpha)) \bar{C}(\alpha)
$$

where $\bar{C}(\alpha):=[C-A(\alpha)] \in \mathbb{R}^{n \times n}$. Since $X$ is the full rank matrix and $p=n-m$ the condition given by (6) is ensured when $\bar{C}(\alpha)$ is invertible. Next, computing determinant of $\bar{C}$ one has: $\operatorname{det} \bar{C}=\operatorname{det} A_{2}$. As a result for $p=n-m$ transversality condition is given by

$$
\forall \alpha \in \mathbb{T}^{n-m}, \operatorname{det} A_{2}(\alpha) \neq 0 .
$$

Relation (11) can be used explicitly to verify if $f$ is a transverse function for the chosen set of parameters, [42].

Selection of a transverse function for the given driftless affine system is not unique. Basically, such a function should satisfy quite general conditions covered by (6) and (8). The constructive method of computation of a transverse function has been proposed in [11]. It is based on the exponential map of non-commuting vector fields with harmonic inputs in order to find implicitly a new direction in the tangent space. This algorithm can be successfully applied to many driftless systems, see for example [43]. When the exponential map cannot be found precisely, one can take advantage of approximated results, [19] or apply coordinate transformations, cf. [13]. However, in many cases formal results of these computations can be seen as an initial candidate for a transverse function. This is due to the fact that one needs to select a proper set of parameters or introduce some modifications to get a required design flexibility. For example, on a Lie group it is possible to find generalized transverse functions (GTF) which can be shrunk to the origin without violating transversality condition. As a result of this feature these functions can be employed to design a controller ensuring asymptotic tracking of some kind of reference trajectories, [12, 42].

2.4. Companion system and decoupling control. The fundamental property of the control method taking advantage of transverse functions is an approximate decoupling of a controllable nonlinear system in spite of kinematic constraints and a possible additive drift. In the considered case one can apply this technique to get an almost linear system.

Assume that $z \in G$ denotes auxiliary configuration. Next, suppose that for $t>0, z(t)$ and configuration $g(t)$ evolve in a sim- ilar way, such that $g(t) \in B_{\varepsilon}(z(t))$, where $B_{r}(p)=\{g \in G: \operatorname{dist}(g$, $p)<r\}$. Let the distance between elements, $z$ and $g$, be determined by transverse function $f$. Then defining an error on Lie group $G$ one can write

$$
z^{-1} g:=f
$$

Alternatively, from (12) one can easily find that

$$
z=g f^{-1} \text {. }
$$

To obtain a more general result we assume that $f$ is dependent not only on $\alpha$, but also on an exogenous variable $\delta \in \mathbb{R}^{l}$ with bounded derivative $\dot{\delta}$. In such a case time derivative of $f$ becomes: $\dot{f}=\frac{\partial f}{\partial \alpha} \dot{\alpha}+\frac{\partial f}{\partial \delta} \dot{\delta}$. Recalling (9) and using basis $X(\cdot)$ one can rewrite $\dot{f}$ as follows: $\dot{f}=X(f)\left(A(\alpha) \dot{\alpha}+A_{\delta}(\alpha) \dot{\delta}\right)$, where $A_{\delta}(\alpha) \in \mathbb{R}^{n \times l}$. Next, computing time derivative of (13) and following analysis presented in [12] we consider the companion system defined by

$$
\dot{z}=X(z) A d^{X}(f)\left(\bar{C}(\alpha) \bar{u}-A_{\delta}(\alpha) \dot{\delta}\right),
$$

where $\bar{u}:=\left[u^{\top} \dot{\alpha}^{\top}\right]^{\top} \in \mathbb{R}^{n}$ is the extended input. Since $X(z) A d^{X}(f) \bar{C}(\alpha) \in \mathbb{R}^{n \times n}$ is the invertible matrix one concludes that $z$ can be changed arbitrarily using bounded input $\bar{u}$. Consequently, system (14) can be seen as an unconstrained system evolving on $G$.

The controller which linearises system (14) can be proposed as follows.

Proposition 1. Decoupling controller. Applying the following control law

$$
\bar{u}=\bar{C}^{-1}(\alpha)\left(A d^{X}\left(f^{-1}\right) X^{-1}(z) w+A_{\delta}(\alpha) \dot{\delta}\right),
$$

where $w \in \mathbb{R}^{n}$ is a new input, to (14) gives fundamental decoupled linear system

$$
\dot{z}=w
$$

Formally, term $w$ in (16) can be regarded as vector field in $\mathfrak{g}$. Computing this field in the basis one can introduce

$$
v:=X^{-1}(z) w
$$

Consequently, using $v$ in (15) yields

$$
\bar{u}=\bar{C}^{-1}(\alpha)\left(A d^{X}\left(f^{-1}\right) v+A_{\delta}(\alpha) \dot{\delta}\right) .
$$

Taking into account that

$$
\bar{C}^{-1}=\left[\begin{array}{cc}
I & -A_{1} A_{2}^{-1} \\
0 & -A_{2}^{-1}
\end{array}\right],
$$

recalling definition of $\bar{u}$ and using (18) one can consider the following augmented dynamics 


$$
\dot{\alpha}=\omega_{\alpha}(\alpha) v+\omega_{\delta}(\alpha) \dot{\delta},
$$

where

$$
\begin{gathered}
\omega_{\alpha}(\alpha):=-A_{2}^{-1}\left[\begin{array}{ll}
0 & I
\end{array}\right] A d^{X}\left(f^{-1}(\alpha)\right), \\
\omega_{\delta}(\alpha):=-A_{2}^{-1}\left[\begin{array}{ll}
0 & I
\end{array}\right] A_{\delta}(\alpha) .
\end{gathered}
$$

This dynamics is essential since it governs evolution of the transverse function. Thus it has a significant impact on evolution of the controlled system and robustness of the closed loopsystem to unmodelled perturbations. Consequently, properties of (20) should be carefully taken into account when application of this control scheme is considered.

\section{Selection of transverse function for a unicycle-like vehicle}

3.1. Model. Here we recall basic definitions and operators which can be employed for a control of the unicycle kinematics, cf. [12]. Assume that $G \cong S E(2)$ is the Lie group with operation given by

$$
g h:=g+\left[\begin{array}{cc}
R\left(g_{\theta}\right) & 0 \\
0 & 1
\end{array}\right] h
$$

where $g, h \in G$ and $R \in S O(2)$ is the planar rotation matrix. In the considered case coordinates of $g$ given by $g_{x}, g_{y}$ and $g_{\theta}$ can be explicitly interpreted, such that $g_{x}$ and $g_{y}$ describe position on the plane, while $g_{\theta}$ denotes orientation. Moreover, neutral element $e=0$ and inverse element satisfies

$$
g^{-1}=-\left[\begin{array}{cc}
R^{\top}\left(g_{\theta}\right) & 0 \\
0 & 1
\end{array}\right] g .
$$

We consider a unicycle-like robot and describe its kinematics using equation (3) assuming that $m=2$, $X_{1}:=\left[\cos g_{\theta} \sin g_{\theta} 0\right]^{\top}$ and $X_{2}:=\left[\begin{array}{lll}0 & 0 & 1\end{array}\right]^{\top}$, while $u_{1}$ and $u_{2}$ denote linear and angular velocity, respectively. It is well known that $X_{1}$ and $X_{2}$ are left-invariant vector fields - hence the unicycle can be globally defined on Lie group $G$. The basis of Lie algebra $\mathfrak{g}$ of group $G$ is determined by three independent left-invariant vector fields $X_{1}, X_{2}$, and $X_{3}:=\left[X_{2}, X_{1}\right]$. To be more precise, we define

$$
X(g):=\left[\begin{array}{lll}
X_{1} & X_{2} & X_{3}
\end{array}\right](g)=\left[\begin{array}{ccc}
\cos g_{\theta} & 0 & -\sin g_{\theta} \\
\sin g_{\theta} & 0 & \cos g_{\theta} \\
0 & 1 & 0
\end{array}\right] .
$$

Additionally, computing adjoint operator one has

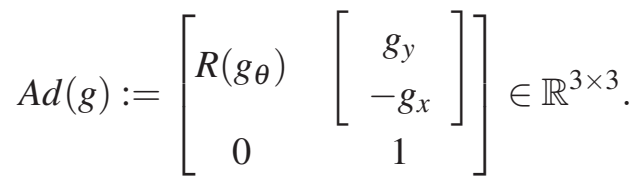

3.2. Selection and parametrization of transverse function. Now we will focus on selection of transverse function $f$ for the unicycle kinematics. We notice that in this case $p=n-m=1$, hence function $f$ evolves on one-dimensional torus $\mathbb{T}^{1}=\mathbb{S}^{1}$. Assume that $f=\left[\begin{array}{lll}f_{x} & f_{y} & f_{\theta}\end{array}\right]^{\top} \in G$ is the transverse function satisfying (6) and (8). Recalling basis $X$ given by (25) and computing $A$ from (9) one obtains

$$
A(\alpha)=\left[\begin{array}{l}
A_{1} \\
A_{2}
\end{array}\right]=\left[\begin{array}{c}
\cos f_{\theta}(\alpha) \frac{\partial f_{x}}{\partial \alpha}+\sin f_{\theta}(\alpha) \frac{\partial f_{y}}{\partial \alpha} \\
\frac{\partial f_{\theta}}{\partial \alpha} \\
\sin f_{\theta}(\alpha) \frac{\partial f_{x}}{\partial \alpha}-\cos f_{\theta}(\alpha) \frac{\partial f_{y}}{\partial \alpha}
\end{array}\right] .
$$

Formally, for the unicycle, one can compute $f$ by analytic means using exponential map, [43]. However, typically an alternative and simpler detailed form of the transverse function, borrowed from the result obtained for a chained system, is used, [12]. We discuss the latter case more thoroughly.

Basically, it is well known that a local homogeneous approximation (cf. [44]) of the unicycle is given by the following I-order two input chained system

$$
\dot{\xi}=\bar{X}_{1} u_{1}+\bar{X}_{2} u_{2}
$$

where $\xi \in \mathbb{R}^{3}$ denotes the configuration, $\bar{X}_{1}=\left[\begin{array}{lll}1 & \xi_{3} & 0\end{array}\right]^{\top}$ and $\bar{X}_{2}=\left[\begin{array}{lll}0 & 0 & 1\end{array}\right]^{\top}$. The transverse function for this system can be found based on the following exponential map

$\bar{f}(\alpha)=\exp \left(\varepsilon_{1} \bar{X}_{1} \sin \alpha+\varepsilon_{2} \bar{X}_{2} \cos \alpha\right)=\left[\begin{array}{c}\varepsilon_{1} \sin \alpha \\ \frac{1}{4} \varepsilon_{1} \varepsilon_{2} \sin 2 \alpha \\ \varepsilon_{2} \cos \alpha\end{array}\right]$,

where $\alpha \in \mathbb{S}^{1}$ and $\varepsilon_{1}$ and $\varepsilon_{2}$ are arbitrarily chosen non-zero parameters.

Taking into account that the unicycle kinematics and the chained system are locally equivalent it implies that function $\bar{f}$ can be seen as a suitable candidate for a transverse function for the former system. Consequently, it can be expected that function (29) is transversal to vector field $X_{1}$ and $X_{2}$ when $\varepsilon_{1}$ and $\varepsilon_{2}$ are selected to be small enough. However, for higher values of $\varepsilon_{1}$ and $\varepsilon_{2}$ preservation of condition (6) is not obvious. Moreover, one can ask if there are some possible modifications of standard form (29) which can be introduced to increase design flexibility. We examine these issues in depth.

In order to make more general statements we introduce new parameter $\varepsilon_{3}$ and consider modified version of (29) as follows

$$
\bar{f}(\alpha)=\left[\begin{array}{c}
\varepsilon_{1} \sin \alpha \\
\bar{\varepsilon}_{3} \sin 2 \alpha \\
\varepsilon_{2} \cos \alpha
\end{array}\right]
$$

where $\bar{\varepsilon}_{3}:=\prod_{i=1}^{3} \varepsilon_{i}$. Notice that in (29) $\varepsilon_{3}=\frac{1}{4}$.

Now we assume that $f=\bar{f}$ and investigate the transversality condition indicated by function $A_{2}$. Namely we look for a feasible selection of $\varepsilon_{1}, \varepsilon_{2}$ and $\varepsilon_{3}$ in order to guarantee that (11) is satisfied. Further, without the lack of generality we assume that $\varepsilon_{1}$ and $\varepsilon_{2}$ are positive and $A_{2}(\alpha)$ should be positive for all $\alpha \in \mathbb{S}^{1}$. Next, we define 


$$
\begin{aligned}
\gamma(\alpha) & :=\frac{1}{\varepsilon_{2}} \sin \left(\varepsilon_{2} \cos \alpha\right) \cos \alpha- \\
& -2 \varepsilon_{3} \cos 2 \alpha \cos \left(\varepsilon_{2} \cos \alpha\right) .
\end{aligned}
$$

and rewrite $A_{2}$ as

$$
A_{2}(\alpha)=\varepsilon_{1} \varepsilon_{2} \gamma(\alpha)
$$

From (31) it follows that $\gamma$ does not depend on $\varepsilon_{1}$. Hence, taking into account (32) one concludes that the transversality condition is satisfied for any $\varepsilon_{1}>0$. Additionally, since $\varepsilon_{2}$ is related to angular measure we restrict upper bound of this parameter to $\pi$.

Further, we consider selection of parameters $\varepsilon_{2}$ and $\varepsilon_{3}$ by analysing function $\gamma$. Using trigonometric identities in (31) we have

$$
\begin{aligned}
\gamma(\alpha)= & \left(\operatorname{sinc}\left(\varepsilon_{2} \cos \alpha\right)-2 \varepsilon_{3} \cos \left(\varepsilon_{2} \cos \alpha\right)\right) \cos ^{2} \alpha+ \\
& +2 \varepsilon_{3} \cos \left(\varepsilon_{2} \cos \alpha\right) \sin ^{2} \alpha,
\end{aligned}
$$

where $\operatorname{sinc}(\sigma):=\frac{\sin \sigma}{\sigma}$ is the analytic function for $\sigma \in \mathbb{R}$. Next, making detailed analysis (see Appendix A.1) one can find feasible values of $\varepsilon_{3}$ for $\varepsilon_{2}$ in the given domain. In Fig. 1 upper bound of $\varepsilon_{3}$ is plotted. In particular for $\varepsilon_{2} \leq \bar{\rho}_{1} \approx 1.139$ parameter $\varepsilon_{3}$ has to be selected such that

$$
\forall \varepsilon_{2} \in\left(0, \bar{\rho}_{1}\right], \varepsilon_{3}<m_{\varepsilon_{3}}=\frac{1}{2} \operatorname{tanc}\left(\varepsilon_{2}\right),
$$

where $\operatorname{tanc}(\sigma):=\frac{\tan (\sigma)}{\sigma}$, while $\sigma \in \mathbb{R}$. It can be noticed that for small $\varepsilon_{2}$ the maximum value of $\varepsilon_{3} \approx 0.5$. This result is not surprising, since it well corresponds to the condition which can be obtained for the approximated system defined by (28). For $\varepsilon_{2} \in\left(\bar{\rho}_{1}, \pi\right]$ there is no closed-analytical formula describing $m_{\varepsilon_{3}}$. Instead, one can follow algorithm presented in Appendix A.1 to find the solution numerically.

Another criterion of selecting parameter $\varepsilon_{3}$ comes from analysis of the control law (15). Considering (19) and (32) one can realize that the value of the lower bound of $\gamma$ is critical. Basically, when $\gamma$ decreases the resultant gain in the open-loop increases and the augmented dynamics becomes sensitive to unmodelled perturbations. Then the control law approaches singularity that can lead to unacceptable performance, [42]. Following this aspect one should prefer to chose $\varepsilon_{3}$ for which the lower bound

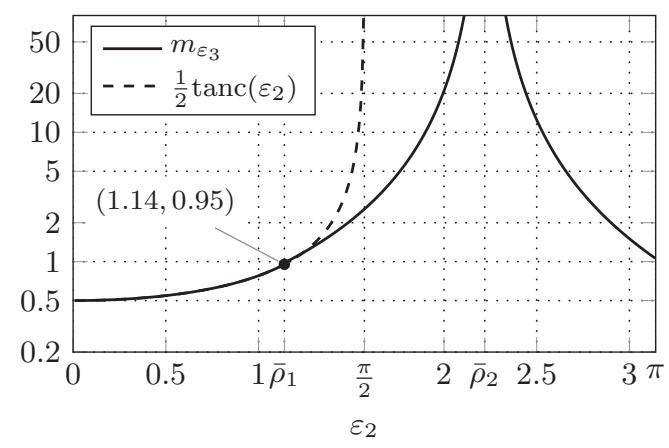

Fig. 1. Upper bound of parameter $\varepsilon_{3}$ for $\varepsilon_{2} \in(0, \pi)$

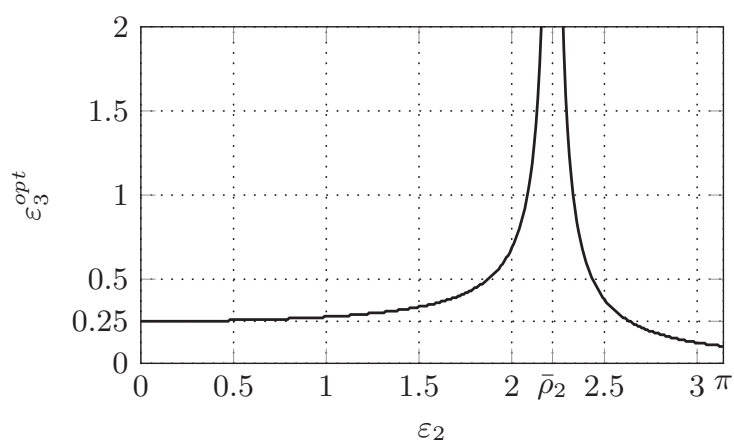

Fig. 2. Optimal value of parameter $\varepsilon_{3}$ maximizing $\inf _{\alpha \in \mathbb{S}^{1}} \gamma(\alpha)$

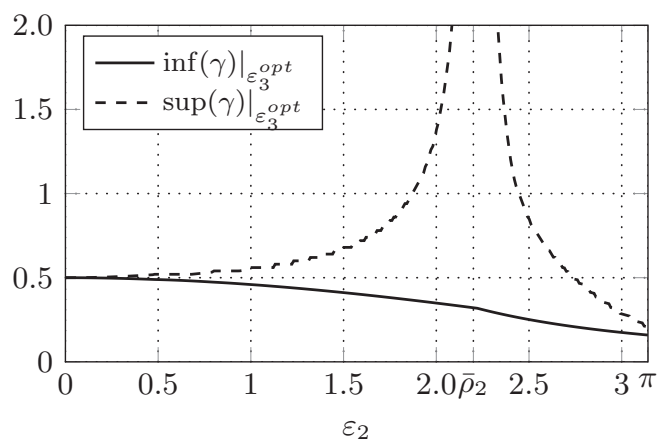

Fig. 3. Global minimum and maximum values of $\gamma(\alpha)$ for the optimal value of parameter $\varepsilon_{3}$

of $\gamma$ is maximised. In Fig. 2 the optimal value of $\varepsilon_{3}$ is presented for the given $\varepsilon_{2}$ while in Fig. 3 the bounds of $\gamma$ for the optimal $\varepsilon_{3}$ is presented. It can be noticed that for a small value of $\varepsilon_{2}$ the optimal selection of $\varepsilon_{3}$ well corresponds to the nominal selection of the transverse function given by (29) for which $\varepsilon_{3}=\frac{1}{4}$. Moreover, it can be proved that $\left(\varepsilon_{2} \rightarrow 0^{+}\right) \Rightarrow\left(\forall \alpha \in \mathbb{S}^{1}, \gamma(\alpha) \rightarrow \frac{1}{2}\right)$, namely for a small $\varepsilon_{2}$ function $\gamma(\alpha)$ becomes independent from $\alpha$ (as is the case of the chained system (28)).

\section{Approximated omnidirectional system}

Recall the definition of auxiliary variable $z \in G$ satisfying (13). It implies that for any $g$ describing configuration of the unicycle one can find the virtual frame characterized by $z=\left[z_{x} z_{y} z_{\theta}\right]^{\top}$, where $z_{x}$ and $z_{y}$ are position coordinates while $z_{\theta}$ stands for orientation - cf. Fig. 4.

Now we recall the decoupling control law defined by (18) taking into account the unicycle model and assuming that $\dot{\delta} \equiv 0$. Consequently, one can specify (18) as follows

$\left[\begin{array}{lll}u_{1} & u_{2} & \dot{\alpha}\end{array}\right]^{\top}=\bar{C}^{-1}(\alpha) A d^{X}\left(f^{-1}\right)\left[\begin{array}{lll}v_{x} & v_{\theta} & v_{y}\end{array}\right]^{\top}$,

where $v_{x}, v_{\theta}$ and $v_{y}$ are velocity components defined in the local frame $X(z)$ (notice that the order of $v$ components is different to the one used to characterize group elements due to the definition of basis $X$ in (25)). These signals can be used to change $z$ in an arbitrary direction. Then $g$ evolves at the neighbourhood 

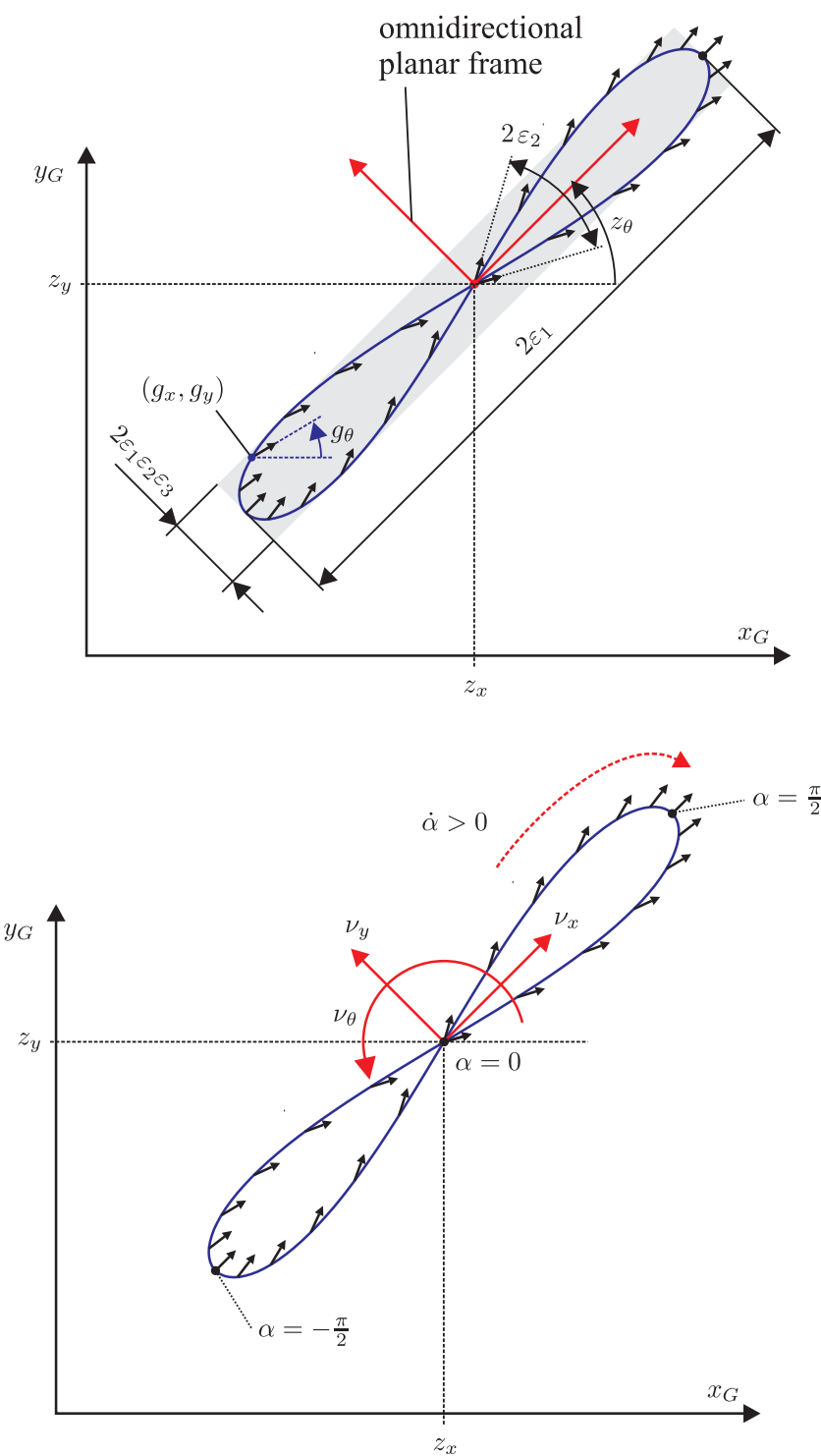

Fig. 4. Geometrical interpretation of the omnidirectional planar frame

of $z$ with the radius dependent on transverse function $f$. Consequently, $g$ can be seen as a closed curve in $G$ parametrized by $\alpha$. In Fig. 4 2D projection of this curve on the plane is presented. This curve can be bounded by a rectangle with the centre placed at $\left(z_{x} z_{y}\right)$ and dimensions dependent on $\varepsilon_{1}, \varepsilon_{2}$ and $\varepsilon_{3}$. Similarly, orientation $g_{\theta}$ can be found for any $\alpha-$ in Fig. 4 this variable is illustrated by short arrows with the corresponding direction. Now taking into account (32) and using (26) the term $\omega_{\alpha}(\alpha)$ from (20) can be calculated as follows

$$
\omega_{\alpha}(\alpha)=-\frac{\bar{\omega}_{\alpha}(\alpha)}{\varepsilon_{1} \varepsilon_{2} \gamma(\alpha)},
$$

where

$$
\begin{aligned}
\bar{\omega}_{\alpha}(\alpha) & =\left[\begin{array}{lll}
0 & 0 & 1
\end{array}\right] A d^{X}\left(f^{-1}(\alpha)\right) \\
& =\left[\begin{array}{lll}
\sin f_{\theta} & -f_{x} \cos f_{\theta}-f_{y} \sin f_{\theta} & \cos f_{\theta}
\end{array}\right](\alpha) .
\end{aligned}
$$

Consequently, the augmented dynamics becomes

$$
\dot{\alpha}=-\frac{1}{\varepsilon_{1} \varepsilon_{2} \gamma(\alpha)} \bar{\omega}_{\alpha}(\alpha) v
$$

4.1. Evolution of augment dynamics. Now we focus on time-evolution of dynamics (38) with respect to input $v$ governing motion of the virtual omnidirectional frame. Components of $v$ can be interpreted from (35) assuming that $f$ approaches $e$ that gives $A d^{X}\left(f^{-1}\right) \approx I$. Then using (19) one obtains

$$
\left[\begin{array}{l}
u_{1} \\
u_{2}
\end{array}\right] \approx\left[\begin{array}{l}
v_{x} \\
v_{\theta}
\end{array}\right]-A_{1} A_{2}^{-1} v_{y}
$$

Hence, it can be concluded that components $v_{x}$ and $v_{\theta}$ are associated with the real kinematic inputs $u_{1}$ and $u_{2}$ of the unicycle, respectively. Alternatively, $v_{y}$ is an infeasible input in the sense that the motion defined by it cannot be directly executed by the unicycle.

Since it is difficult to formulate precise conclusions for the general case, we consider particular cases in the sequel. In order to do this we define fundamental motion patterns (manoeuvres) collected in Table 1. We distinguish two kinds of patterns - feasible, which can be realized straightforward by the unicycle and infeasible which violate nonhnolonomic constraints. To simplify analysis it is assumed that input $v$ is constant at least during some time interval.

Table 1

Basic motion patterns

$\begin{array}{clccc}\text { Case } & \text { Motion } & v_{x} & v_{\theta} & v_{y} \\ \text { I } & \text { feasible straight line } & \pm & 0 & 0 \\ \text { II } & \text { pure rotation } & 0 & \pm & 0 \\ \text { III } & \text { feasible arc } & \pm & \pm & 0 \\ \text { III } & \text { infeasible straight line } & \pm & 0 & \pm \\ \text { IV } & \text { infeasible arc } & \pm & \pm & \pm\end{array}$

Let $\alpha_{0}$ be an equilibrium point of system (38), namely

$$
\bar{\omega}_{\alpha}\left(\alpha_{0}\right) v=0
$$

In order to investigate the stability of (38) we introduce the following positive definite function

$$
V:=\frac{1}{2} \tilde{\alpha}^{2}
$$

where $\tilde{\alpha}:=\alpha-\alpha_{0}$ and compute its time derivative $\dot{V}$. Substituting (38) to $\dot{V}$ one has

$$
\dot{V}=-\frac{1}{\varepsilon_{1} \varepsilon_{2} \gamma\left(\tilde{\alpha}+\alpha_{0}\right)} \tilde{\alpha} \bar{\omega}_{\alpha}\left(\tilde{\alpha}+\alpha_{0}\right) v .
$$


4.2. Case I: feasible straight motion. Assume that $v_{x} \neq 0$ while $v_{\theta}$ and $v_{y}=0$. Computing $\bar{\omega}_{\alpha} v$, in view of (37), one has

$$
\bar{\omega}_{\alpha} v=\sin f_{\theta} v_{x}=\sin \left(\varepsilon_{2} \cos \alpha\right) v_{x} .
$$

The equilibrium $\alpha_{0} \in\left\{-\frac{\pi}{2}, \frac{\pi}{2}\right\}$. It can be found that for $\varepsilon_{2} \in(0, \pi)$ the following relationship holds

$$
\forall \tilde{\alpha} \in(-\pi, 0) \cup(0, \pi), \dot{V}<0 \text { for } \alpha_{0}=-\frac{\pi}{2} \operatorname{sign}\left(v_{x}\right) .
$$

Consequently, it follows that $\alpha_{0}=-\frac{\pi}{2} \operatorname{sign}\left(v_{x}\right)$ is the locally asymptotically stable equilibrium point.

4.3. Case II: pure rotation. Let $v_{x}=v_{y}=0$ and $v_{\theta} \neq 0$ which implies from (37) that

$\bar{\omega}_{\alpha} v=-\left(f_{x} \cos f_{\theta}+f_{y} \sin f_{\theta}\right) v_{\theta}=-\kappa_{1}(\alpha) \varepsilon_{1} \sin \alpha v_{\theta}$,

where

$$
\kappa_{1}(\alpha):=\cos \left(\varepsilon_{2} \cos \alpha\right)+2 \varepsilon_{2} \varepsilon_{3} \cos \alpha \sin \left(\varepsilon_{2} \cos \alpha\right)
$$

It can be shown (see Appendix A.2) that

$$
\forall \alpha \in \mathbb{S}^{1}, \varepsilon_{3}>-\left(2 \varepsilon_{2} \tan \varepsilon_{2}\right)^{-1}, \kappa_{1}(\alpha)>0 .
$$

Hence, in view of (46), the equilibrium point satisfies $\alpha_{0}=0$. Consequently, using (42) one can prove that

$\forall \tilde{\alpha} \in(-\pi, 0) \cup(0, \pi), \dot{V}<0$ for $\alpha_{0}= \begin{cases}\pi & \text { if } v_{\theta}>0 \\ 0 & \text { if } v_{\theta}<0\end{cases}$

4.4. Case III: feasible motion along arc. In this case we have $v_{x}=$ const $\neq 0, v_{\theta}=$ const $\neq 0$ and $v_{y}=0$. Then it follows from (37) that

$$
\begin{aligned}
\bar{\omega}_{\alpha} v & =\sin f_{\theta} v_{x}-\left(f_{x} \cos f_{\theta}+f_{y} \sin f_{\theta}\right) v_{\theta}= \\
& =\kappa_{2}\left(\alpha, v_{x}, v_{\theta}\right),
\end{aligned}
$$

where

$$
\kappa_{2}\left(\alpha, v_{x}, v_{\theta}\right):=\sin \left(\varepsilon_{2} \cos \alpha\right) v_{x}-\kappa_{1}(\alpha) \varepsilon_{1} \sin \alpha v_{\theta}
$$

Considering equation $\kappa_{2}\left(\alpha, v_{x}, v_{\theta}\right)=0$ one concludes that there is no closed formula to describe its roots in the general case. Basically, the value of the equilibrium point is dependent on the relation between inputs $v_{x}$ and $v_{\theta}$, as well as the selection of parameters $\varepsilon_{1}, \varepsilon_{2}$ and $\varepsilon_{3}$.

One can find an approximate solution assuming that $\varepsilon_{2}$ selected is small enough such that $\kappa_{1}$ satisfies

$$
\kappa_{1}(\alpha) \approx 1+2 \varepsilon_{2}^{2} \varepsilon_{3} \cos ^{2} \alpha \approx 1
$$

and $\kappa_{2}$ can be approximated as follows

$$
\begin{aligned}
& \kappa_{2}\left(\alpha, v_{x}, v_{\theta}\right) \approx \bar{\kappa}_{2}\left(\alpha, v_{x}, v_{\theta}\right):= \\
& :=\varepsilon_{2} \cos (\alpha) v_{x}-\varepsilon_{1} \sin (\alpha) v_{\theta} .
\end{aligned}
$$

Roots of $\bar{\kappa}_{2}\left(\alpha, v_{x}, v_{\theta}\right)=0$ satisfy

$$
\tan \alpha_{0}=\frac{\varepsilon_{2} v_{x}}{\varepsilon_{1} v_{\theta}}
$$

Next, using (53) and investigating the sign of $\dot{V}$ defined by (42) one can compute value of the asymptotically stable equilibrium point as follows

$$
\alpha_{0}=\operatorname{atan} 2\left(-\varepsilon_{2} v_{x},-\varepsilon_{1} v_{\theta}\right),
$$

where $\operatorname{atan} 2(\cdot, \cdot)$ stands for the four-quadrant inverse tangent function.

Alternatively, it is possible to find sets of equilibrium points by solving (49) numerically for the given parameters. The results illustrated in Fig. 5 are obtained assuming that $v_{x}=N \cos \beta$, $v_{\theta}=N \sin \beta$, where $N>0$ and $\beta \in(-\pi, \pi]$, for selected ratio $\varepsilon_{1} / \varepsilon_{2}$ and $\varepsilon_{3}=\frac{1}{4}$.
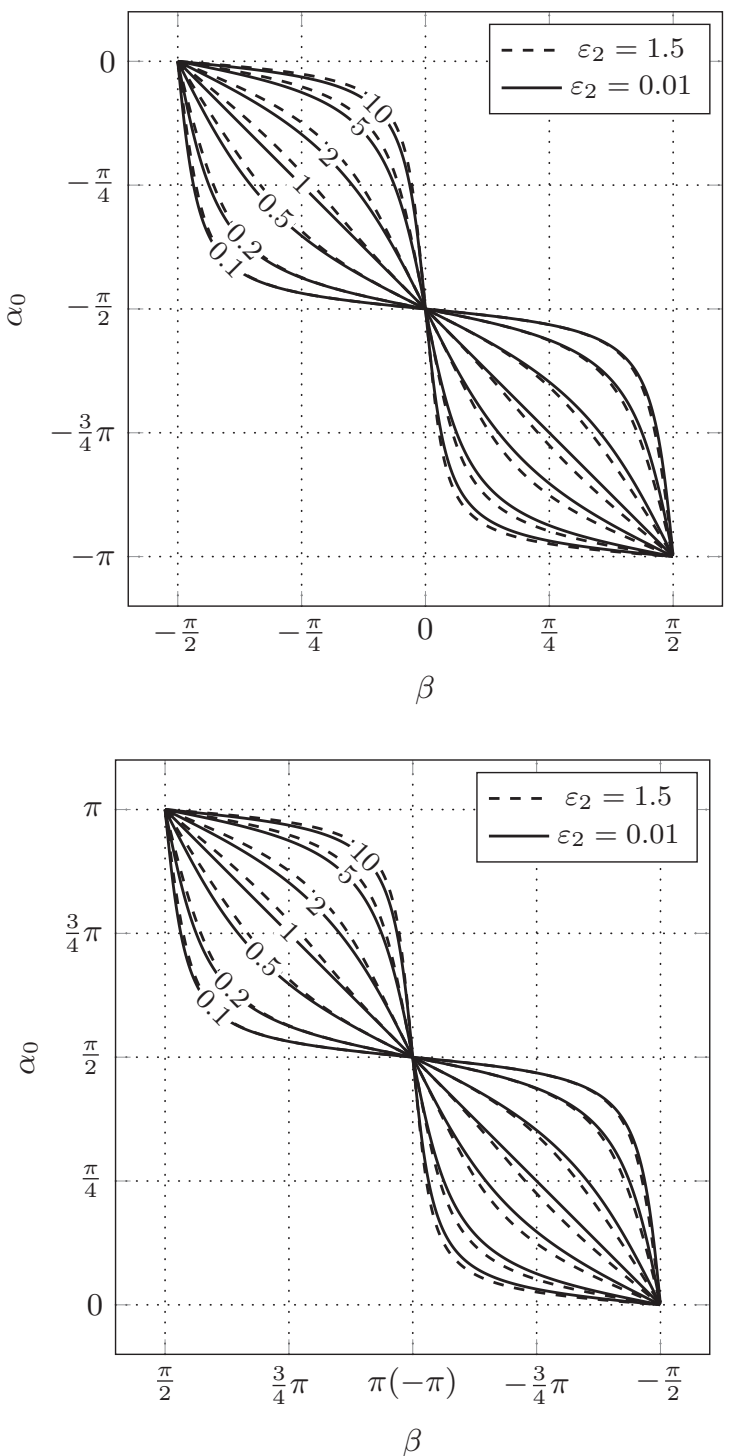

Fig. 5. Set of equilibrium points in Case III for the given inputs $v_{x}=N \cos \beta, v_{\theta}=N \sin \beta$ and various selections of parameter $\varepsilon_{1}$ and $\varepsilon_{2}$ and $\varepsilon_{3}=\frac{1}{4}$ - the coefficients on curves denote ratio $\varepsilon_{1} / \varepsilon_{2}$ 


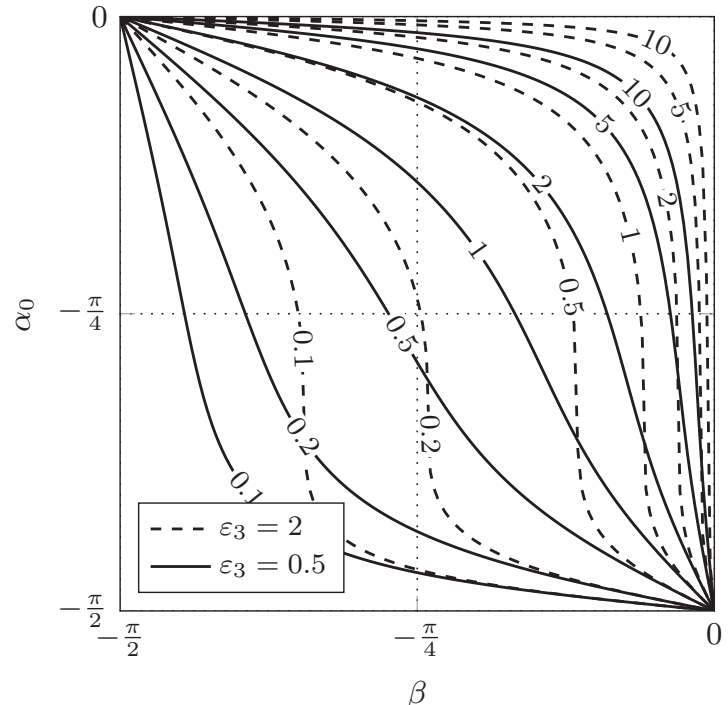

Fig. 6. Set of equilibrium points in Case III for the given inputs $v_{x}=N \cos \beta, v_{\theta}=N \sin \beta$ and various selections of parameter $\varepsilon_{1}$ and $\varepsilon_{3}$ with $\varepsilon_{2}=1.5$ - the coefficients on curves denote ratio $\varepsilon_{1} / \varepsilon_{2}$

It can be observed that the value of equilibrium point $\alpha_{0}$ for small $\varepsilon_{2}$ well corresponds to the approximated analytical solution defined by (54). Substituting the assumed inputs in (54) one obtains: $\alpha_{0}:=\operatorname{atan} 2\left(-\varepsilon_{2} \cos \beta,-\varepsilon_{1} \sin \beta\right)=\operatorname{atan} 2\left(\varepsilon_{2} \sin \left(-\beta-\frac{\pi}{2}\right)\right.$, $\left.\varepsilon_{1} \cos \left(-\beta-\frac{\pi}{2}\right)\right)$. Hence, for $\varepsilon_{1}=\varepsilon_{2}$ one easily concludes that $\alpha_{0}=-\beta-\frac{\pi}{2}$. In Fig. 5 this case is illustrated by almost straight lines denoted by '1'. For the higher value of $\varepsilon_{2}$ one can notice that parameter $\varepsilon_{3}$ affects the value of the equilibrium point considerably. Then the approximated result (54) is no longer accurate - cf. Fig. 6 .

4.5. Case IV - infeasible linear motion. Assuming that $v_{x}=$ const $\neq 0, v_{y}=$ const $\neq 0$ and recalling (37) allows one to write

$$
\bar{\omega}_{\alpha} v=\sin f_{\theta} v_{x}+\cos f_{\theta} v_{y}
$$

Clearly, the equilibrium point can be found when there is a solution to equation: $\tan \left(f_{\theta}(\alpha)\right)=-v_{y} / v_{x}$. Assuming that $\varepsilon_{2}<\frac{\pi}{2}$ this condition can be satisfied when

$$
\sup _{\alpha}\left|f_{\theta}(\alpha)\right|<\arctan \left|\frac{v_{y}}{v_{x}}\right| .
$$

Basically, it means that an equilibrium can be achieved when

$$
\left|\frac{v_{y}}{v_{x}}\right|<\tan \varepsilon_{2} .
$$

Following this result, one concludes that the augmented dynamics (38) has the equilibrium even for some kind of infeasible motion pattern. This is an interesting issue which comes from the self adapting property of transverse functions. Basically, it means that there is some tolerance to infeasible input $v$ for which the augmented dynamics is stable and no peri- odically cycles are observed. Moreover, this tolerance can be easily modified by parameter $\varepsilon_{2}$. For smaller $\varepsilon_{2}$ the unicycle is controlled such that difficult directions are realized in an oscillatory way. When $\varepsilon_{2}$ increases the controller attenuates this behaviour since more change of orientation is permissible. In particular, for $\varepsilon_{2}=\frac{\pi}{2}$ function $\tan \varepsilon_{2}$ becomes unbounded and for any infeasible straight stationary motion an equilibrium point can be found. A similar conclusion can be made for $\varepsilon_{2}>\frac{\pi}{2}$. It follows that for a higher value of $\varepsilon_{2}$ orientation of the unicycle is adjusted properly in order to establish the desired change of position coordinates.

To make more detailed analysis it is assumed that $v_{x}=N \cos \beta$ and $v_{y}=N \sin \beta$ where $N>0$. Using these inputs in (55) allows one to rewrite (38) as follows

$$
\dot{\alpha}=-\frac{N}{\varepsilon_{1} \varepsilon_{2} \gamma(\alpha)}\left(\sin f_{\theta} \cos \beta+\cos f_{\theta} \sin \beta\right) .
$$

Next, we define scaled time $\tau:=N \frac{t}{\varepsilon_{1} \varepsilon_{2}}$ and apply it to (58). Additionally, using trigonometric identities one obtains the following normalized dynamics

$$
\frac{d \alpha}{d \tau}=-\frac{1}{\gamma(\alpha)} \sin \left(f_{\theta}+\beta\right) .
$$

Integrating (59) numerically one can determine the mean normalized frequency of oscillation for the given values of $\varepsilon_{2}, \varepsilon_{3}$ and $\beta$. The results of this computation are presented in Fig. 7 for $\beta \in\left[0, \frac{\pi}{2}\right)$. It can be seen that when $\beta$ is close to zero the feasible input $v_{x}$ dominates and no oscillation is observed. When $\beta$ becomes higher than $\varepsilon_{2}$ the dynamics (59) starts to generate a periodic cycle.

Having normalized frequency $F_{n}$ one can compute real frequency of oscillation as follows

$$
F_{\text {osc }}=\frac{N}{\varepsilon_{1} \varepsilon_{2}} F_{n} .
$$

Hence, it is possible to predict a response of the controller for the assumed inputs $v_{x}$ and $v_{y}$. This is an important issue when the oscillatory response of the controller should be limited. Such a requirement is necessary when a practical implementation of the controller is considered.

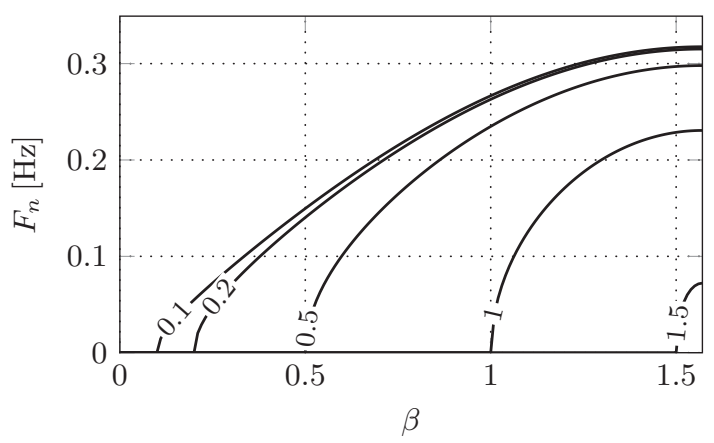

Fig. 7. Normalized mean frequency of oscillation for infeasible linear motion computed for the selected value of $\varepsilon_{2}$ and $\varepsilon_{3}=\frac{1}{4}$ 
4.6. Case $\mathbf{V}$ - infeasible motion along arc. This case is the most complicated and it can be seen as a superposition of Case III and Case IV. Using again (37) and (46) one obtains

$$
\bar{\omega}_{\alpha} v=\sin f_{\theta} v_{x}+\cos f_{\theta} v_{y}-\kappa_{1}(\alpha) \varepsilon_{1} \sin \alpha v_{\theta}
$$

Similarly as in the Case IV one can study an approximate solution assuming that $\varepsilon_{2}$ selected is small enough such that $\kappa_{1} \approx 1$ and $\sin \left(\varepsilon_{2} \cos \alpha\right) \approx \varepsilon_{2} \cos \alpha$. Then one can define

$$
\bar{\omega}_{\alpha} v \approx \kappa_{3}(\alpha):=\varepsilon_{2} \cos \alpha v_{x}+v_{y}-\varepsilon_{1} \sin \alpha v_{\theta}
$$

and consider the following equation: $\kappa_{3}(\alpha)=0$. The solution to this equation can be found only when $v_{y}$ is not significant. To be more precise one has

$$
\varepsilon_{2} \cos \alpha v_{x}+v_{y}-\varepsilon_{1} \sin \alpha v_{\theta}=0 .
$$

Defining $v_{x}=N \varepsilon_{1} \sin \beta$ and $v_{\theta}=N \varepsilon_{2} \cos \beta$, using these signals in (63) and taking advantage of trigonometric identities gives

$$
\varepsilon_{1} \varepsilon_{2} N \sin (\alpha-\beta)=v_{y} .
$$

In order to find a solution to (64) one concludes that

$$
\left|v_{y}\right|<\varepsilon_{1} \varepsilon_{2} N=\varepsilon_{2} \sqrt{v_{x}^{2}+\left(\frac{\varepsilon_{1}}{\varepsilon_{2}}\right)^{2} v_{\theta}^{2}} .
$$

Basically, it can be found that inequality (65) for $v_{\theta}=0$ corresponds to (57), since $\varepsilon_{2} \approx \tan \varepsilon_{2}$. It confirms that when the input in the infeasible direction does not exceed some threshold system (38) evolves to an equilibrium. Otherwise, the augmented dynamics generates a periodic cycle similar to the one observed in the case IV.

4.7. Extension to non-constant parameters of the transverse function. The studied decoupling controller (35) is designed assuming that parameters of transverse function $f$ are constant coefficients. However, in order to facilitate tuning of the controller (for example to decrease frequency of oscillations for an infeasible motion pattern) one can employ feed-forward term $A_{\delta}(\alpha) \delta$ from (18). In the considered case variable $\delta$ can be used to characterize parameters of $f$. To be more precise, one can assume that $\delta:=\left[\begin{array}{lll}\varepsilon_{1} & \varepsilon_{2} & \varepsilon_{3}\end{array}\right]^{\top}$. Consequently, it is possible to change these parameters freely (assuming that the transversality condition holds and $\dot{\delta}$ is bounded) without affecting the position and orientation of the omnidirectional frame described by $z$. Then, one can adapt distance between $z$ and $g$ taking into account additional requirements and to improve performance of the controller.

\section{Application of the controller for velocity-based control in task space}

For many feedback planning algorithms taking advantage of potential functions it is assumed that a robot is not subject to phase constraints and is fully actuated. Consequently, every direction in the phase space can be executed independently using a relatively simple motion controller. Then it is possible to use the basic idea of attracting and repulsive vector fields designed based on the gradient of potential functions. In such a case the main problem is to construct a potential in order to avoid local minima.

Here we consider the feedback motion planning algorithm assuming that obstacles are isolated from each other. Moreover, a local sensing zone is supposed - namely each obstacle can interact with a robot locally in an external region. The robot is modelled as a virtual particle without velocity constraints.

Basically, the considered problem can be stated as follows.

Problem 1. Feedback navigation problem. Let $p \in \mathbb{R}^{2}$ be the coordinate of the point robot on the plane with holes (obstacles). The robot kinematics is given by

$$
\dot{p}=w
$$

where $w=\left[\begin{array}{ll}w_{1} & w_{2}\end{array}\right]^{\top}$ is input. Assume that there exists a feedback navigation algorithm $\Omega(p)$ which provides a bounded and differentiable velocity field in $\mathbb{R}^{2}$ such that trajectory $p(t)$ remains in a free space. Design a controller for a planar world with star obstacles for a differentially driven robot with the unicycle kinematic such that position of the robot follows $p(t)$ with bounded error while its orientation maintains in the desired range.

5.1. Environment description for virtual omnidirectional robot. Consider open workspace $\mathscr{W}=\mathbb{R}^{2}$ with set of $l$ obstacles $\mathscr{W} \mathscr{O}:=\sum_{i=1}^{k} \mathscr{W} \mathscr{O}_{i}$ and define free space $\mathscr{W}_{\text {free }}:=\mathscr{W} \backslash \mathscr{W} \mathscr{O}$. Assume that a real robot for the given configuration $g \in G \cong \mathrm{SE}(2)$ occupies area $\mathscr{W}_{R}(g)$. Accordingly, it is possible to find the free configuration space defined by

$$
\mathscr{Q}_{\text {free }}:=\left\{g \in G: \mathscr{W}_{R}(g) \cap \mathscr{W} \mathscr{O}=\emptyset\right\} .
$$

Then a motion planning/control problem in a geometrically constrained workspace can be described in terms of the configuration variables. Following this idea we consider a virtual omnidirectional robot with coordinates given by $p:=\left[\begin{array}{ll}z_{x} & z_{y}\end{array}\right]^{\top} \in \mathbb{R}^{2}$ and $z_{\theta} \in \mathbb{S}^{1}$. Recalling (13) one can notice that the space occupied by the robot is dependent on $z$ and $\alpha$. Therefore an additional safe space has to be reserved around each obstacle. Consequently, we define $i^{\text {th }}$ virtual obstacle which satisfies

$$
\begin{aligned}
\mathscr{Z} \mathscr{O}_{i}:= & \left\{p \in \mathscr{W}: \forall z_{\theta}, \alpha \in \mathbb{S}^{1},\right. \\
& \left.\mathscr{W}_{R}(z f(\alpha)) \cap \mathscr{W} \mathscr{O}_{i} \neq \emptyset\right\} .
\end{aligned}
$$

To make a simpler model we introduce the enlarged virtual obstacle $\mathscr{O}_{i}$ such that

$$
\mathscr{Z} \mathscr{O}_{i} \subset \mathscr{O}_{i} \subset \mathscr{W}
$$

and $\mathscr{O}_{i}$ has a star-like shape, cf. [28]. Here it should be emphasized that since the upper norm of $f$ is known from the de- 


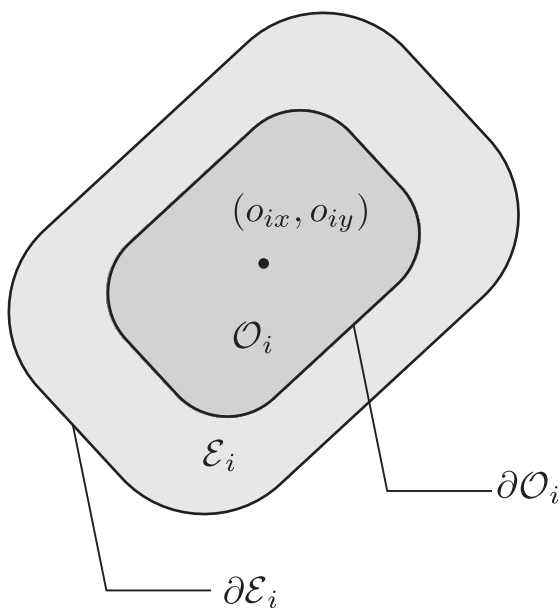

Fig. 8. Example of a star obstacle surrounded by a local interaction zone

sign the desired safety margin can be precisely determined. Additionally, we consider external zone $\mathscr{E}_{i}$ in order to define a transient region where the given obstacle can interact with the robot, cf. Fig. 8 .

Similarly to (67) we introduce virtual free configuration space

$$
\mathscr{Z}_{\text {free }}:=\mathscr{W} \backslash \bigcup_{i=1}^{l} \mathscr{O}_{i}
$$

Definition 2. Let $\mathscr{F}$ be a differentiable star defined by

$$
\mathscr{F}:=\left\{p \in \mathbb{R}^{2}: F(p)-1 \leq 0\right\}
$$

where $F(p)$ satisfies

1. $F(p) \in C^{2}$ in the set $B_{0}(\rho) \supset \mathscr{F}$,

2. $F(p)$ is positive definite and has global minimum at $p=0$.

To be more precise we define

$$
\mathscr{O}_{i}:=\left\{p \in \mathbb{R}^{2}: F_{o_{i}}\left(p-o_{i}\right) \leq 1\right\}
$$

and

$$
\mathscr{E}_{i}:=\left\{p \in \mathbb{R}^{2}: F_{o_{i}}\left(p-o_{i}\right) \leq F_{e_{i}}\left(p-o_{i}\right) \leq 1\right\},
$$

where $o_{i}=\left[o_{i x} o_{i y}\right]^{\top} \in \mathbb{R}^{2}$ denotes the coordinates of the centre of both regions while $F_{o_{i}}(p)$ and $F_{e_{i}}(p)$ satisfy conditions given by Definition 2 .

5.2. Obstacle avoidance for circular obstacles. Next, we consider the case when $\mathscr{O}_{i}=\mathscr{O}_{i}^{c}$ is a circular (disk) obstacle with radius $r_{i}$. Then $F_{o_{i}}\left(p-o_{i}\right):=\frac{1}{r_{i}^{2}}\left\|p-o_{i}\right\|^{2}$ and the repulsive potential of the obstacle can be defined as

$$
V_{o_{i}}^{c}(p)= \begin{cases}\frac{r_{i}^{2}}{\left\|p-o_{i}\right\|^{2}-r_{i}^{2}} & \text { if }\left\|p-o_{i}\right\|>r_{i} \\ 0 & \text { otherwise }\end{cases}
$$

From (74) it follows that $V_{o_{i}}$ is unbounded on obstacle boundary $\partial \mathscr{O}_{i}^{c}$.
Computing gradient of $V_{o_{i}}$ gives

$$
\frac{\partial V_{o_{i}}^{c}(p)}{\partial p}= \begin{cases}-\frac{2}{r_{i}^{2}}\left(V_{o_{i}}^{c}(p)\right)^{2}\left(p-o_{i}\right)^{\top} & \text { if }\left\|p-o_{i}\right\|>r_{i} \\ 0 & \text { otherwise }\end{cases}
$$

Assuming that the interaction area for $i^{\text {th }}$ obstacle is restricted to set $\mathscr{E}_{i}$ and taking into account result (75) one can propose the following repulsive vector field

$w_{o_{i}}(p):= \begin{cases}-\left(F_{e_{i}}\left(p-o_{i}\right)-1\right)^{\delta} \frac{\partial V_{o_{i}}^{c}(p)^{\top}}{\partial p} & \text { if } F_{e_{i}}\left(p-o_{i}\right)<1 \\ 0 & \text { otherwise }\end{cases}$

where $\delta=2,4, \ldots$ is an even integer and $F_{e_{i}}$ can be interpreted as a scaling function which limits the interaction area (notice that gradient $\frac{\partial V_{o_{i}}^{c}(p)}{\partial p}$ is non zero at the exterior of the obstacle).

Taking into account all $l$ circular obstacles one can propose the following resultant repulsive field

$$
w_{o}(p)=\sum_{i=1}^{l} w_{o_{i}}(p)
$$

Next, we introduce a radially symmetric vector field which smoothly attracts the point robot to the goal and define

$$
w_{a}(p)=-p+p_{d},
$$

where $p_{d} \in \mathscr{Z}_{\text {free }} \backslash \bigcup_{i=1}^{l} \mathscr{E}_{i}$ denotes position of the goal.

The resultant navigation field is composed of the attractive and the repulsive fields as follows

$$
w(p)=k_{a} w_{a}(p)+k_{o} w_{o}(p)
$$

where $k_{a}$ and $k_{o}$ are positive coefficients used for a tuning purpose. Recalling that interaction areas of obstacles do not overlap it is clear that for any $p$ one can analyse an isolated system composed of one circular obstacle and the goal. In such a case it is well known that for a radially symmetric attractor no local minima appear and the only critical points (when field $w$ is degenerated to zero) are the saddle point and the global minimum at $p_{d},[45]$.

5.3. Obstacle avoidance for star obstacles. Considering a more general model of the environment with star obstacles, the local minima issue can occur even for one convex obstacle, [45]. Hence, the potential and the corresponding repulsive vector field have to be computed in a different way. For example, one can take advantage of a coordinate transformation in order to reshape a star to a disk. Following the algorithm proposed by Rimon and Koditschek [28] we consider the following map in the neighbourhood of a star:

$$
p_{c}=\beta(p):=r \frac{p-o_{i}}{\left\|p-o_{i}\right\|} \sqrt{F_{o_{i}}\left(p-o_{i}\right)}+o_{i},
$$

where $r$ is the radius of the disc contained in the given star. This transformation maps the boundary of the star on the boundary of the disk with radius $r$. Next, based on (74) potential $V\left(p_{c}\right)$ is computed. Correspondingly, gradient of $V\left(p_{c}\right)$ is determined 


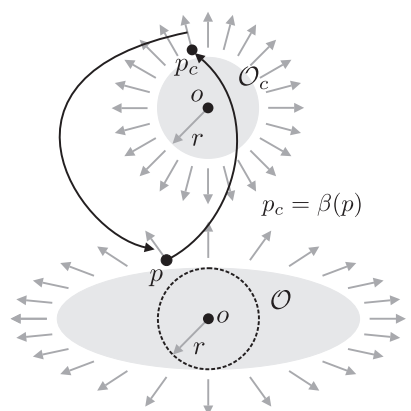

Fig. 9. Interpretation of deformation of a star shape to a disc and lifting of vector field near the obstacle

based on (75) and then it is lifted from $p_{c}$ to $p$. In order to attenuate the repulsive field on the exterior of $\mathscr{E}_{i}$ one can use function $F_{e i}$ similarly as in the case of the disk obstacle, cf. (76).

\subsection{Integration of navigation algorithm with motion con-} troller. Now assuming that $w(p)$ is a bounded vector field (it is guaranteed when $p \notin \partial \mathscr{O}_{i}$, where $\left.i=1, \ldots, l\right)$ one can combine navigation algorithm (79) with control algorithm (35). Basically, components of vector fields $w$ are given in the inertial frame, while inputs $v_{x}$ and $v_{y}$ are defined in the local omnidirectional frame. Recalling (17) in the considered case one can write

$$
v=X^{-1}(z) w^{*}
$$

where $z=\left[p^{\top} z_{\theta}\right]^{\top} \in G$ and $w^{*}=\left[w^{\top} w_{\theta}\right]^{\top} \in \mathbb{R}^{3}$, while $w_{\theta}$ is an additional component.

So far only the position coordinates, $p=\left[z_{x} z_{y}\right]^{\top}$ of the virtual robot have been taken into account. However, the assumed model of the omnidirectional robot consists of virtual frame with orientation $z_{\theta}$. As a result of the decoupling - cf. (16) - it is possible to treat $z_{\theta}$ as an independent variable which can be controlled by input $w_{\theta}$ (or $v_{\theta}$ as a result of (81)). To be more precise, assume that $\phi \in \mathbb{S}^{1}$ is a desired bounded differentiable trajectory and define the following tracking error

$$
e_{\phi}:=z_{\theta}-\phi
$$

Taking time derivative of $e_{\phi}$ and recalling that $\dot{z}_{\theta}=v_{\theta}$ the following open-loop dynamics can be considered

$$
\dot{e}_{\phi}=v_{\theta}-\dot{\phi},
$$

where $\dot{\phi}$ is a bounded term by definition.

Consequently, in order to ensure that $e_{\phi} \rightarrow 0$ one can propose the following classic stabilizer.

Proposition 2. Let $\dot{\phi}$ be a bounded term. Then the auxiliary control law defined as follows

$$
v_{\theta}=-k_{\phi}\left(z_{\theta}-\phi\right)+\dot{\phi}
$$

where $k>0$, applied to linear system $\dot{z}_{\theta}=v_{\theta}$ ensures exponential stability of (83).
Now we consider limitations of arbitrary selection of $\phi$. In spite of the fact that the virtual frame is omnidirectional one should be aware that the motion task is executed by the system with nonholonomic constraints. Recalling analysis of the basic motion patterns discussed in Section 4.1 it is clear that infeasible directions can be only approximated. When the constraints are strongly violated (it is dependent on the selected parameters) the unicycle moves in an oscillatory way. Clearly, there is a contradiction between the execution of an infeasible motion and the attenuation of the zig-zag motion response.

Taking into account these aspects one can usually prefer to use simple motion patterns which are almost feasible. In order to do this one can use a heading controller and try to steer virtual omnidirectional robot in order to maintain its direction defined by $z_{\theta}$ to be compatible with navigation vector field $w$. Following this idea one can design function $\phi$ as follows

$$
\phi:=\operatorname{atan} 2\left(w_{y}, w_{x}\right)
$$

where $\|w\|>0$. In this case time derivative of $\phi$ satisfies

$$
\dot{\phi}=\frac{w^{\top}}{\|w\|^{2}} J \dot{w}
$$

with $J:=\left[\begin{array}{cc}0 & 1 \\ -1 & 0\end{array}\right]$ being a skew-symmetric matrix. Here, the controller given by (84) can be applied conditionally. Using (86) one can see that $\dot{\phi}$ is dependent on time derivative of navigation vector field $w$. This might be considered as a serious limitation in practice since $\dot{w}$ describes a linear acceleration which is not trivial to be computed (or measured) efficiently. Taking into account this issue one can use a simplified version of controller by neglecting the term $\dot{\phi}$ in (84). In such a case the closed loop dynamics becomes: $\dot{e}_{\phi}=-k_{\phi} e_{\phi}-\dot{\phi}$, where $\dot{\phi}$ is not compensated. Thus it is not guaranteed that error $e_{\phi}$ tends to zero - instead it remains bounded for the bounded $\dot{\phi}$. Such a result is sufficient since for the considered motion controller the precise tracking of $\phi$ is not necessary. This can be seen as an important advantage of this control strategy.

Another issue is related to the computation of $\phi$. From (85) it follows that $\phi$ can be determined when $\|w\|>0$. In order to cope with the singularity at $w=0$ one can consider the following simplified heading controller

$$
v_{\theta}=-k_{\phi} \tanh \|w\|\left(z_{\theta}-\phi\right)
$$

Since $\phi$ and $z_{\theta}$ are bounded variables in $\mathbb{R}$ it is clear that: $\|w\| \rightarrow 0 \Rightarrow \tanh \|w\|\left(z_{\theta}-\phi\right)=0$. As a result for a weak navigation field (when $\|w\|$ is small) the heading control is attenuated. Hence, the discussed method allows one to achieve a relatively high robustness to unmodelled dynamics and measurement noise without losing basic navigation properties.

5.5. Extension to trajectory tracking. Basically, the navigation vector field (79) can be also applied for a non stationary goal. However, it does not guarantee an asymptotic tracking result with respect to the omnidirectional frame due to the un- 
compensated velocity of the target. Hence, one can extend the navigation algorithm as follows

$$
w(p)=k_{a} w_{a}(p)+k_{o} w_{o}(p)+\dot{p}_{d},
$$

where $\dot{p} \in \mathbb{R}^{2}$ denotes bounded components of the goal velocity expressed in the inertial frame. It should be emphasized that obstacle avoidance is still guaranteed since $w_{o}(p)$ is unbounded on obstacles boundaries and the repulsive field is dominant.

5.6. Tuning of the motion controller in the navigation task. In subsection 4.7 it is stated that parameters of the transverse function of the considered motion controller can be changed during its operation. This possibility can be employed for the navigation task especially when infeasible motion patterns are required.

The key idea of the navigation method considered here is based on control of the virtual omnidirectional frame instead of the nonholonomic robot directly. Taking into account a safe navigation requirement one can easily find that a control precision can be locally relaxed when the distance between the robot and obstacles is significant. Assuming that the robot moves in a low cluttered area it is possible to execute various manoeuvres in extensive way without collisions. In such a case one can safely increase the distance between the omnidirectional frame and the robot frame in order to improve robustness of the closed-loop to unmodelled dynamics and to attenuate possible oscillatory motions. Conversely, when the robot navigates through a cluttered region it is required that the robot should be close enough to the virtual one. Thus, function $f$ should be made as small as needed (recall that the non zero distance between the robot and the navigation frame can be taken into account by increasing dimensions of modelled obstacles).

Here we propose an idea how to scale the transverse function. It turns out that the investigated navigation method provides a convenient measure determining the occupancy level of the environment close to the robot. This measure is based on the repulsive potential. Assume that $V_{o_{i}}\left(g_{x}, g_{y}\right)$ denotes the non-negative potential of $i^{\text {th }}$ obstacle determined for the current position of the robot. This potential can be computed using algorithm discussed in sections 5.2 and 5.3. Aggregating all repulsive potentials one has: $V_{o}=\sum_{i=1}^{l} V_{o_{i}}\left(g_{x}, g_{y}\right)$. In order to improve robustness of the algorithm we define the filtered potential $V_{o}^{f}$ satisfying

$$
\dot{V}_{o}^{f}=-k_{f}\left(V_{o}^{f}-V_{o}\right),
$$

where $1 / k_{f}>0$ denotes the time constant.

Term $V_{o}^{f}$ can be used to define parameters of the transverse function. We consider the particular proposition of tuning assuming that only parameter $\varepsilon_{1}$ is changed. This parameter scales components $f_{x}$ and $f_{y}$, cf. (30), and determines the Euclidean distance between position coordinates $\left(z_{x}, z_{y}\right)$ and $\left(g_{x}, g_{y}\right)$. Later on it is assumed that $\varepsilon_{1}$ is defined by the following formula

$$
\varepsilon_{1}=\frac{\varepsilon_{1 \max }-\varepsilon_{1 \mathrm{~min}}}{k_{\varepsilon_{1}} V_{o}^{f}+1}+\varepsilon_{1 \mathrm{~min}},
$$

where $k_{\varepsilon_{1}}>0$ is a positive constant, while $\varepsilon_{1 \min }$ and $\varepsilon_{1 \max }$ denote the positive lower and upper bound of $\varepsilon_{1}$, respectively. It can be easily concluded that when the robot is away from any obstacle $V_{o}^{f} \rightarrow 0$ and $\varepsilon_{1} \rightarrow \varepsilon_{1 \max }$. When the robot approaches boundary of any obstacle $V_{o}^{f}$ increases and $\varepsilon_{1} \rightarrow \varepsilon_{1 \mathrm{~min}}$.

Time derivative of $\varepsilon_{1}$ can be easily determined from (89) and (90). Next, computing term $A_{\delta}(\alpha) \dot{\delta}$ one can use $\dot{\varepsilon}_{1}$ in the motion controller defined by (18).

It is worth noting that the presented tuning algorithm can be further extended in order to modify other parameters of the transverse function. In particular, the detailed propositions are dependent on specific requirements formulated for the given motion task as well as implementation issues met in practice.

\section{Simulation and experimental results\}}

6.1. Simulation of the omnidirectional frame. First, we make simulation in Matlab/Simulink environment of the control system with motion controller (35). Parameters of the transverse function are set as follows: $\varepsilon_{1}=0.05$ and $\varepsilon_{2}=\frac{\pi}{6}$, while $\varepsilon_{3} \in\{0.05,0.25,0.5\}$. Velocity input $v$ applied to the omnidirectional frame is chosen as

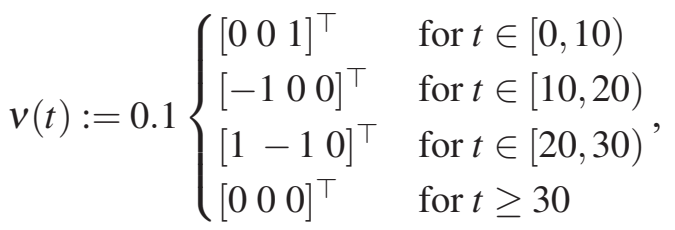

while initial configuration of the unicycle satisfies $g(0)=0$.

Consequently, only the second motion stage defined for $t \in[10,20)$ is feasible for the considered nonholonomic kinematics. Other segments are highly infeasible and they are executed using an oscillatory-like pattern as can be observed from Fig. 10. Comparing the obtained curves $\left(g_{x}, g_{y}\right)$ one concludes

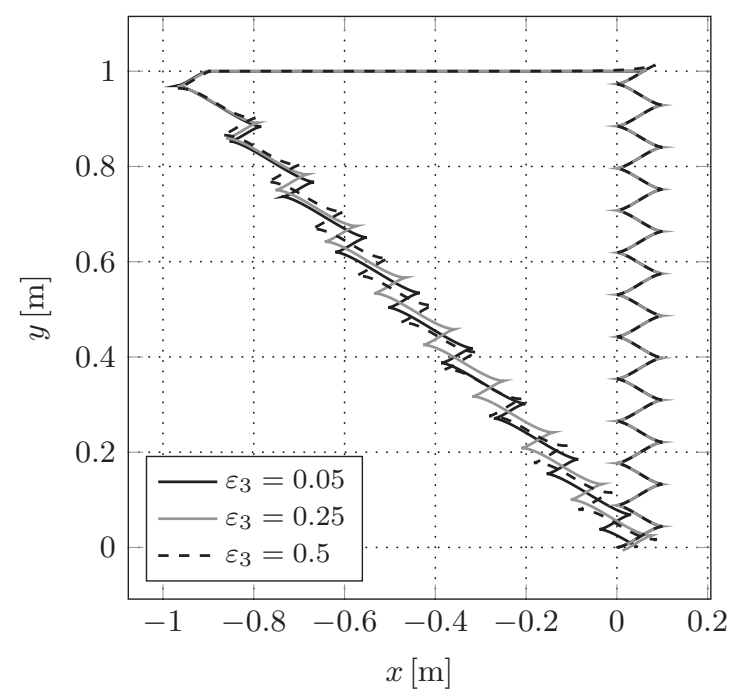

Fig. 10. Position path $\left(g_{x}, g_{y}\right)$ obtained for the selected values of $\varepsilon_{3}$ 
that parameter $\varepsilon_{3}$ does not affect the path shape considerably. In particular, the first two segments are almost invariant to the selection of $\varepsilon_{3}$. Some difference can be noticed for the last segment - then the number of manoeuvres increases slightly for a higher value of $\varepsilon_{3}$.
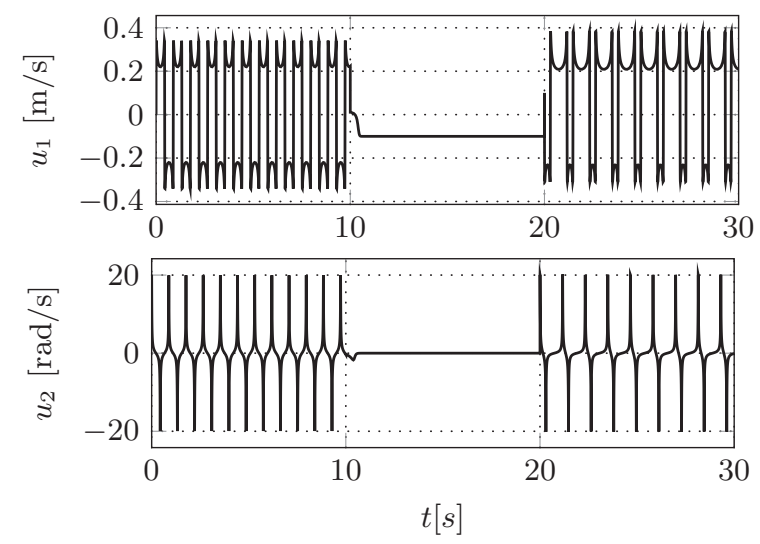

Fig. 11. Inputs $u_{1}$ and $u_{2}$ obtained for $\varepsilon_{3}=0.05$
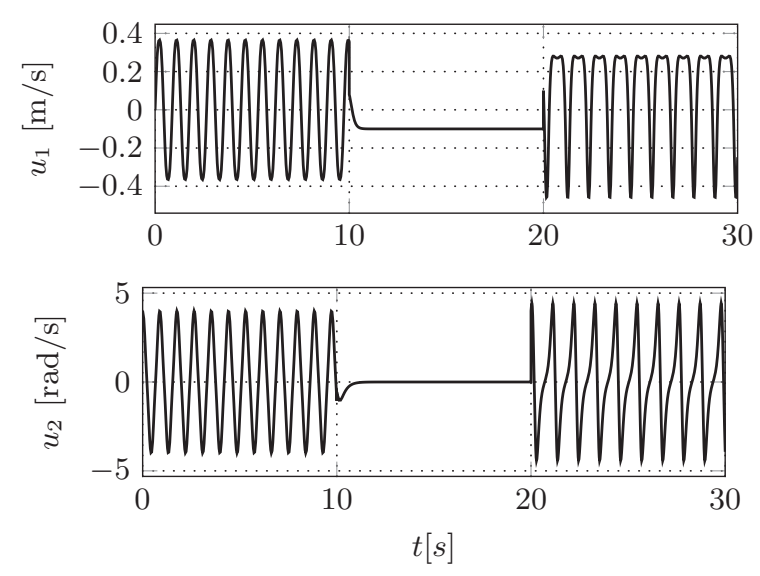

Fig. 12. Inputs $u_{1}$ and $u_{2}$ obtained for $\varepsilon_{3}=0.25$
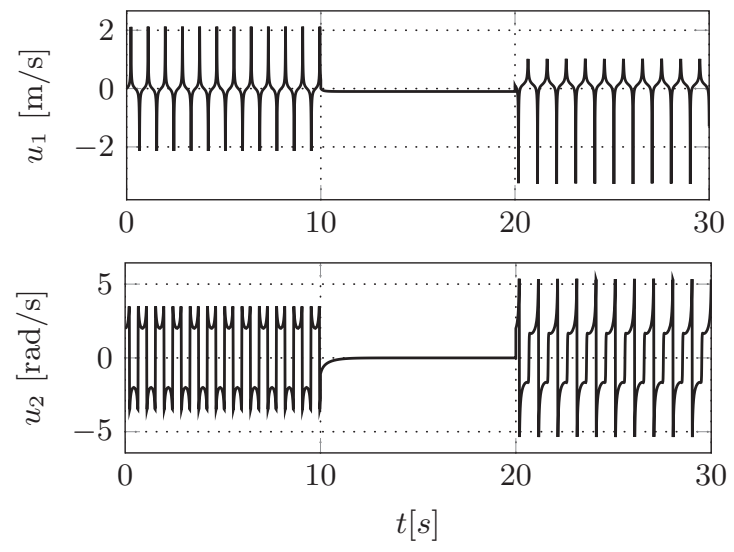

Fig. 13. Inputs $u_{1}$ and $u_{2}$ obtained for $\varepsilon_{3}=0.5$
Otherwise, parameter $\varepsilon_{3}$ modifies input signals considerably - cf. Figs. 11-13. More smooth time plots are observed when $\varepsilon_{3} \approx 0.25$. This correspond to the optimal selection of $\varepsilon_{3}$ discussed in Section 3 - cf. Fig. 2. Computing integrals of squared inputs - see Table 2 - it can be confirmed that the same motion action (with respect to the path shape and the mean velocity) is executed with different energy expenditure.

Table 2

Comparison of the control effort computed for the given set of parameter $\varepsilon_{3}$ for the scenario considered in the simulation

$\begin{array}{cccc}\text { Parameter } \varepsilon_{3} & \int_{0}^{30} u_{1}(t)^{2} d t & \int_{0}^{30} u_{2}(t)^{2} d t & \int_{0}^{30} u(t)^{\top} u(t) d t \\ 0.05 & 1.34 & 327 & 328 \\ 0.25 & 1.56 & 133 & 135 \\ 0.5 & 4.27 & 112 & 116\end{array}$

6.2. Experimental results of the navigation controller. In order to verify properties of the motion controller with the proposed navigation method, experimental work was conducted using a laboratory two-wheeled robot MTracker [46] with wheels radius $r_{w}=0.025 \mathrm{~m}$ and the distance between the wheels $b=0.145 \mathrm{~m}$. The algorithm was implemented in $\mathrm{C}++$ code and run on a PC which sends velocity commands to the on-board DSP controller 50 times per second. The robot was localized using the odometry. The wheel velocities were saturated using smooth scaling algorithm (in order to suppress hard nonlinearities). Additionally, time scaling was applied to guarantee proper integration of the augmented dynamics.

The obstacles were modelled numerically as smooth superellipses, [47] and their dimensions were increased to include the robot diameter as well as an additional distance margin defined by the transverse function, cf. (68). The motion controller parameters were selected as follows: $\varepsilon_{1}=0.05$, $\varepsilon_{2}=0.5, \varepsilon_{3}=0.3$, while initial condition $\alpha(0)=-\frac{\pi}{2}$. The parameters of the navigation controller given by (79) were set as: $k_{a}=k_{a}(p)=\frac{0.125}{\|w(p)\|+0.25}$ and $k_{o}=0.2$. The given formula describing $k_{a}$ allows one to limit magnitude of the attractive field when the robot is far away from the goal.

- Experiment 1 (E1): motion with the robot heading alignment

- Experiment 2 (E2): motion with simultaneous and independent rotation

- Experiment 3 (E3): motion with simultaneous and independent rotation with the adaptation of the transverse function

- Experiment 4 (E4): tracking of the circular trajectory with the robot heading alignment

- Experiment 5 (E5): motion of the robot between obstacles with overlapping external zones with the heading alignment

- Experiment 6 (E6): motion of the robot between obstacles with overlapping external zones with almost constant orientation

In experiment E1 the static goal with coordinates $p_{d}=\left[\begin{array}{ll}4 & 2.5\end{array}\right]^{\top}$ is taken into account. The maximum values of wheel velocities are limited to $10 \mathrm{rad} / \mathrm{s}$. It is assumed that the obstacles including their external zones are isolated from each other - see Fig. 14a. 
In order to avoid oscillatory motion of the robot the auxiliary heading controller (87) is applied with $k_{\phi}=20$. The motion task is executed properly - the goal is achieved without any collision within about 35 s. From Fig. 15a one can see the navigation

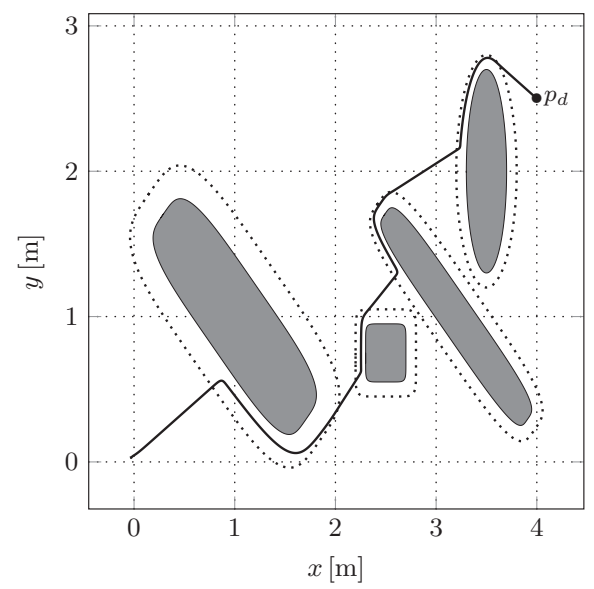

(a) Experiment E1: path $\left(z_{x}, z_{y}\right)$

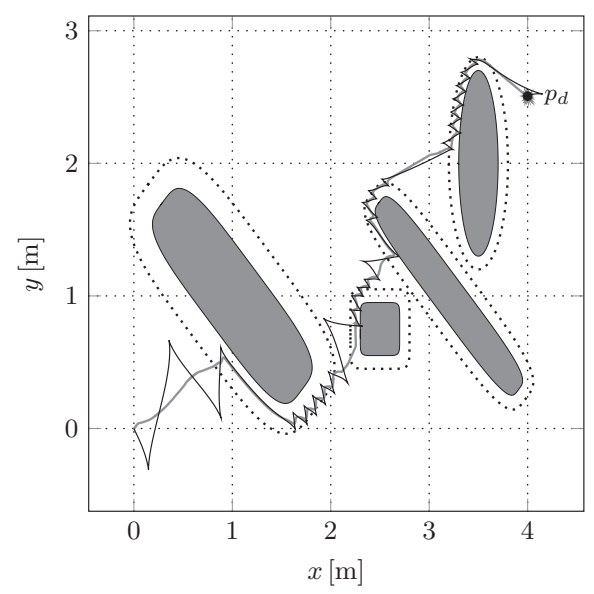

(c) Experiment E3: path $\left(z_{x}, z_{y}\right)$ (gray line) and $\left(g_{x}, g_{y}\right)$ (black line)

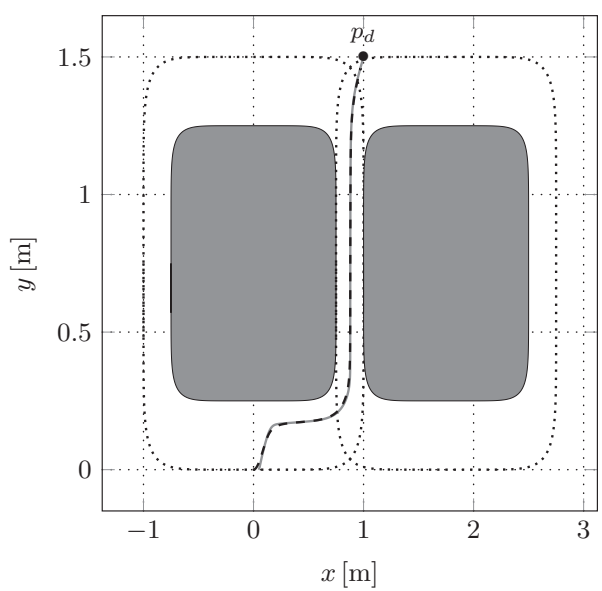

(e) Experiment E5: path $\left(z_{x}, z_{y}\right)$ (gray line) and $\left(g_{x}, g_{y}\right)$ (dashed black line) components describing linear velocity in the local omnidirectional frame. One can notice that in spite of using the heading controller term $v_{y}$ is not precisely attenuated. In particular, at about 18 th second $v_{y}$ becomes significant for a short time in-

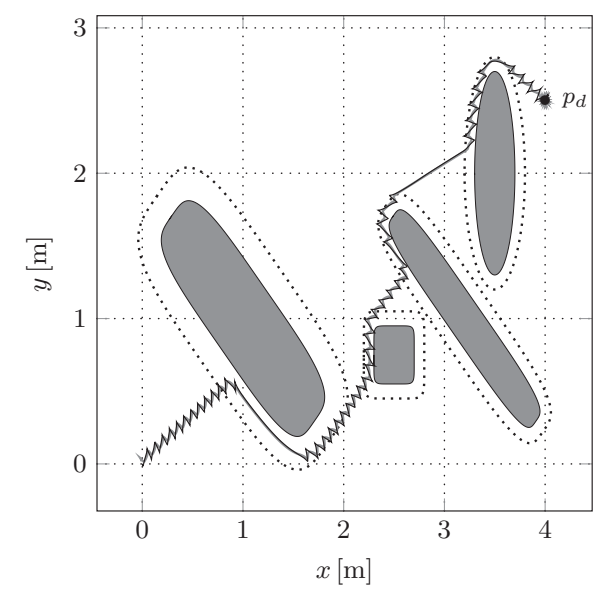

(b) Experiment E2: path $\left(z_{x}, z_{y}\right)$ (gray line) and $\left(g_{x}, g_{y}\right)$ (black line)

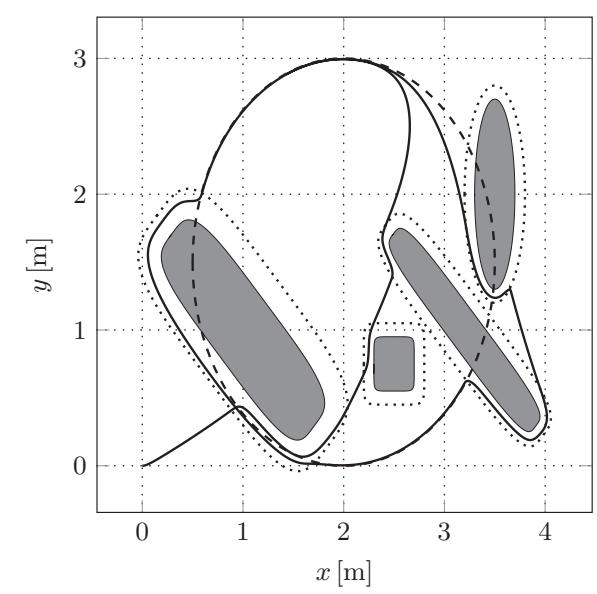

(d) Experiment E4: path $\left(g_{x}, g_{y}\right)$ (black line) and $\left(p_{d_{x}}, p_{d_{y}}\right)$ (dashed black line)

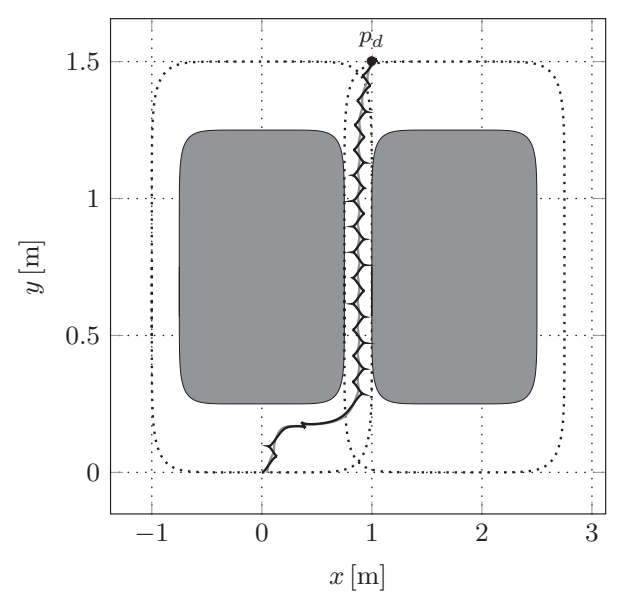

(f) Experiment E6: path $\left(z_{x}, z_{y}\right)$ (gray line) and $\left(g_{x}, g_{y}\right)$ (dashed black line)

Fig. 14. Results of experiments E1-E6: the environment structure and position paths 


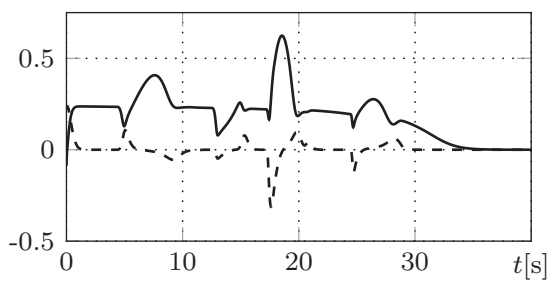

(a) Navigation inputs $v_{x}$ (solid) and $v_{y}$ (dashed) in $[\mathrm{m} / \mathrm{s}]$

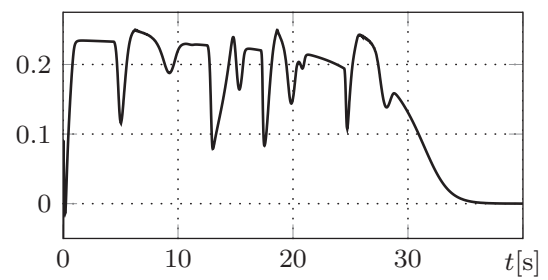

(d) Linear velocity $u_{1}$ of the unicycle in $[\mathrm{m} / \mathrm{s}]$

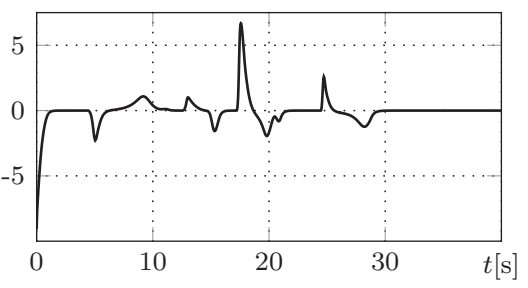

(b) Auxiliary angular input $v_{\theta}[\mathrm{rad} / \mathrm{s}]$

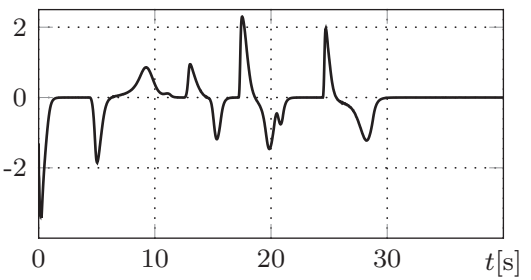

(e) Angular velocity $u_{2}$ of the unicycle in $[\mathrm{rad} / \mathrm{s}]$

Fig. 15. Results of experiment E1

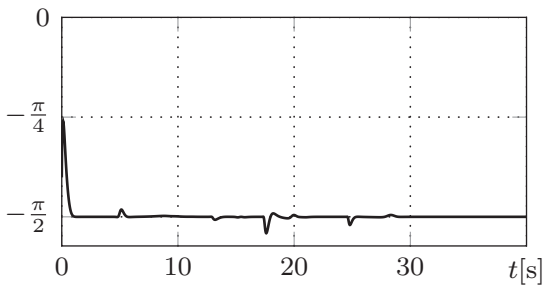

(c) Time evolution of auxiliary variable $\alpha$ in $[\mathrm{rad}]$

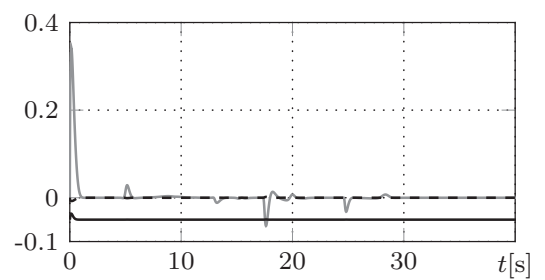

(f) Time evolution of the TF: $f_{x}$ (black line), $f_{y}$ (dashed black line), $f_{\theta}$ (gray line) terval. This is the result of a significant change of $\phi$ which is not followed accurately by $z_{\theta}$ due to signal saturation and absence of $\dot{\phi}$ in (87). In spite of it the tracking precision is sufficient to avoid an oscillatory response of the closed-loop system for the chosen parameter $\varepsilon_{2}$. It can be concluded that input $v$ is almost feasible such that oscillatory motion patterns are not employed. From Fig. 15d it can be observed that the robot moves forward with positive linear velocity during execution of the motion task. As a result the augmented dynamics is not excited and variable $\alpha$ stays close to $-\pi / 2$ (as indicated from stability of augmented dynamics considered in Section 4.1) and components of transverse function $f$ do not change significantly - cf. Figs. 15c and f. Control inputs $u_{1}$ and $u_{2}$ are smoothly saturated in order to meet the assumed bounds. As a result the magnitudes of these signals are scaled down in comparison to the magnitudes of auxiliary inputs $v_{x}$ and $v_{\theta}-\mathrm{cf}$. Figs. $15 \mathrm{a}, \mathrm{b}, \mathrm{d}$ and e.

The next experiment, E2, was conducted assuming that the omnidirectional frame moves toward the goal and rotates with angular velocity $0.075 \mathrm{rad} / \mathrm{s}$. The upper bound of the angular velocities of the robot wheels were increased to $30 \mathrm{rad} / \mathrm{s}$. This scenario could correspond to an exploration task when the robot is supposed to observe a surrounding environment from different angles. In this case the heading controller is not used. As a result the navigation field in general is highly infeasible for the unicycle - from Fig. 16a it can be seen that $v_{y}$ achieves significant values. From Fig. $14 \mathrm{~b}$ it can be no-

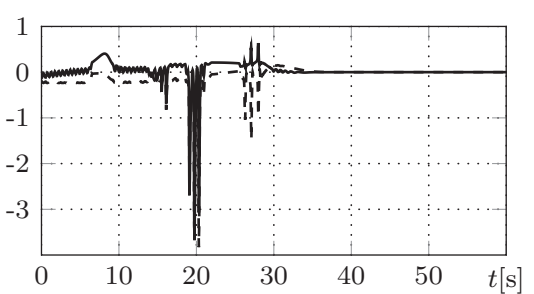

(a) Navigation inputs $v_{x}$ (solid) and $v_{y}$ (dashed) in $[\mathrm{m} / \mathrm{s}]$

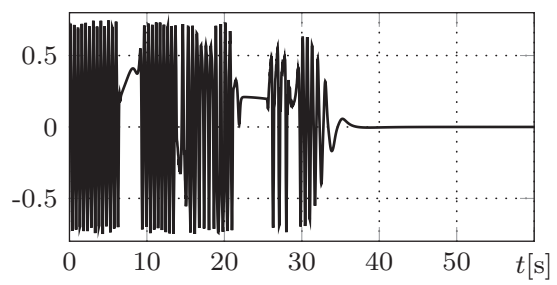

(d) Linear velocity $u_{1}$ of the unicycle in $[\mathrm{m} / \mathrm{s}]$

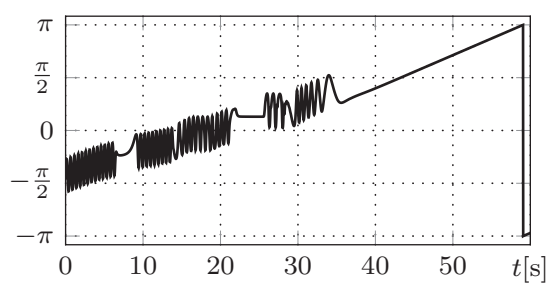

(b) Orientation of the unicycle $g_{\theta}$ in $[\mathrm{rad}]$

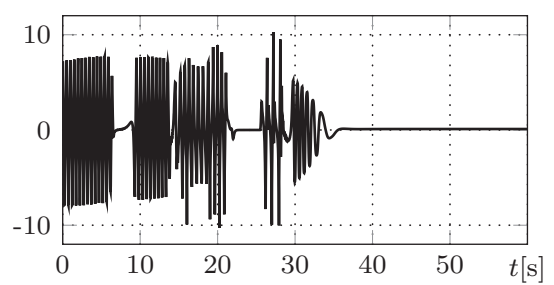

(e) Angular velocity $u_{2}$ of the unicycle in $[\mathrm{rad} / \mathrm{s}]$

Fig. 16. Results of experiment E2

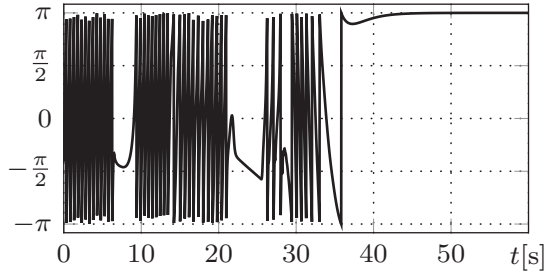

(c) Time evolution of auxiliary variable $\alpha$ in $[\mathrm{rad}]$

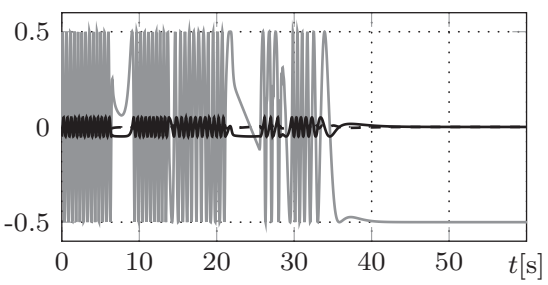

(f) Time evolution of the TF: $f_{x}$ (black line), $f_{y}$ (dashed black line), $f_{\theta}$ (gray line) 
ticed that a zig-zag motion pattern is executed by the robot in order to approximate the path given by $\left(z_{x}, z_{y}\right)$. Comparing results of experiments E1 and E2 illustrated in Figs. 14a and $14 \mathrm{~b}$, respectively, one concludes that the position of the omnidirectional frame evolves in a similar way in spite of the fact that nonholonomic constraints are highly violated - the robot orientation increases in an approximate sense during experiment E2 - cf. Fig. 16b. However, in order to approximate infeasible directions highly oscillatory input signals are produced - see Figs. 16d and e. This is a result of excitation of the augmented dynamics which is forced to generate peri- odic cycles with the frequency mainly dependent on value of $v_{y}-\mathrm{cf}$. Figs. $16 \mathrm{c}$ and $\mathrm{f}$.

Basically, in experiment E3 the same scenario is considered as in the previous case. The main modification is the application of adapting scheme discussed in Section 5.6. To be more precise, it was assumed that parameter $\varepsilon_{1}$ is a time varying function bounded by $\varepsilon_{1 \text { min }}=0.05$ and $\varepsilon_{1 \max }=0.4$. Other parameters of the algorithm defined by (89) and (90) were selected as: $1 / k_{f}=0.2$ and $k_{\varepsilon_{1}}=20$. Comparing results of experiments E2 and E3 one can easily notice that highly oscillatory manoeuvres are realized near obstacles - cf. Fig. 14c. In the case when the

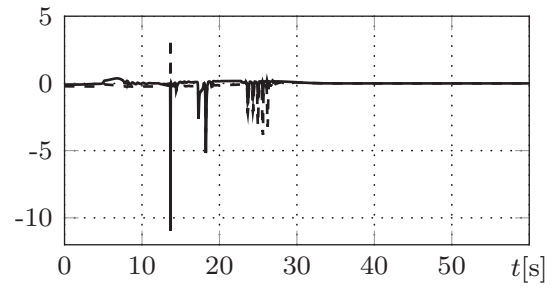

(a) Navigation inputs $v_{x}$ (solid) and $v_{y}$ (dashed) in $[\mathrm{m} / \mathrm{s}]$

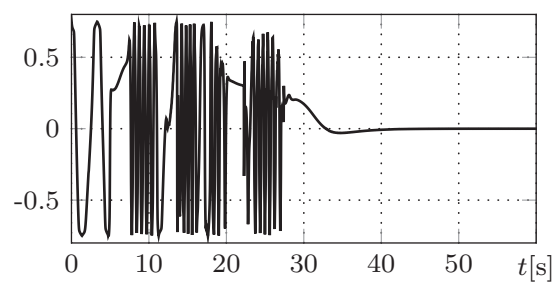

(d) Linear velocity $u_{1}$ of the unicycle in $[\mathrm{m} / \mathrm{s}]$

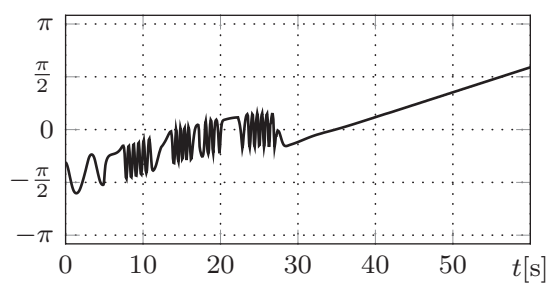

(b) Orientation of the unicycle $g_{\theta}$ in [rad]

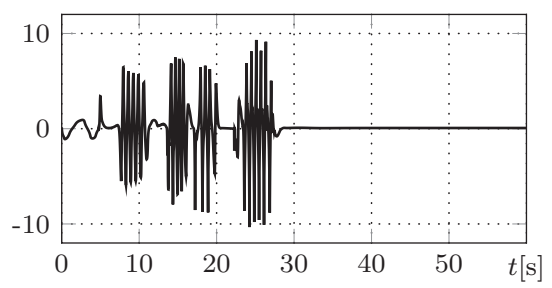

(e) Angular velocity $u_{2}$ of the unicycle in $[\mathrm{rad} / \mathrm{s}]$

Fig. 17. Results of experiment E3

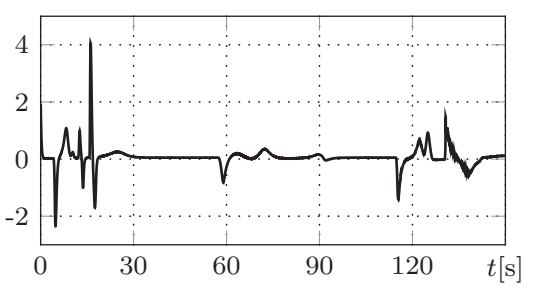

(b) Auxiliary angular input $v_{\theta}[\mathrm{rad} / \mathrm{s}]$

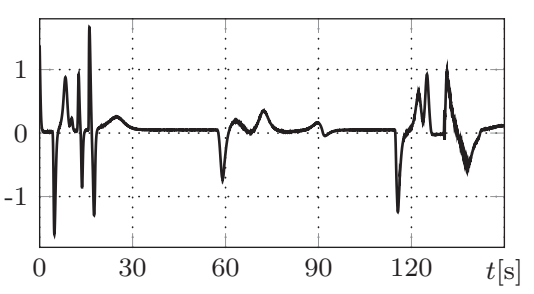

(e) Angular velocity $u_{2}$ of the unicycle in $[\mathrm{rad} / \mathrm{s}]$

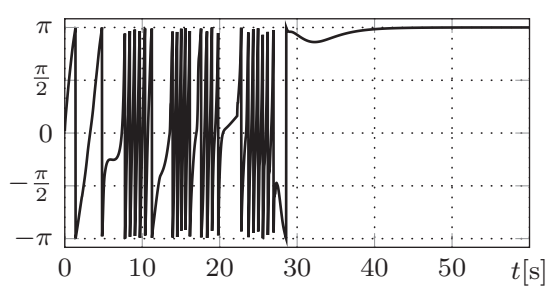

(c) Time evolution of auxiliary variable $\alpha$ in $[\mathrm{rad}]$

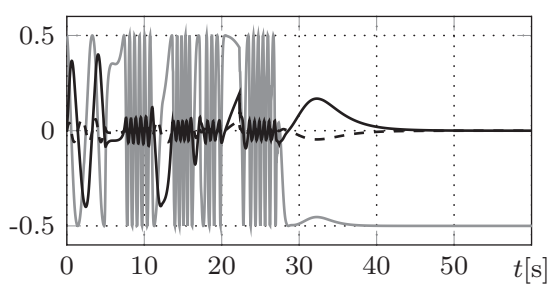

(f) Time evolution of the TF: $f_{x}$ (black line), $f_{y}$ (dashed black line), $f_{\theta}$ (gray line)

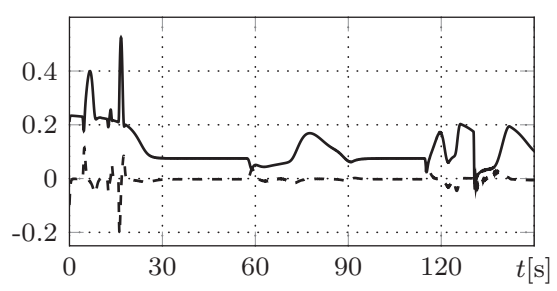

(a) Navigation inputs $v_{x}$ (solid) and $v_{y}$ (dashed) in $[\mathrm{m} / \mathrm{s}]$

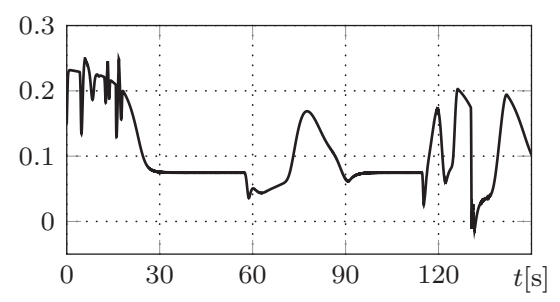

(d) Linear velocity $u_{1}$ of the unicycle in $[\mathrm{m} / \mathrm{s}]$

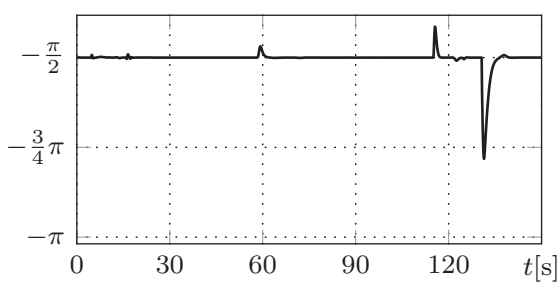

(c) Time evolution of auxiliary variable $\alpha$ in $[\mathrm{rad} / \mathrm{s}]$

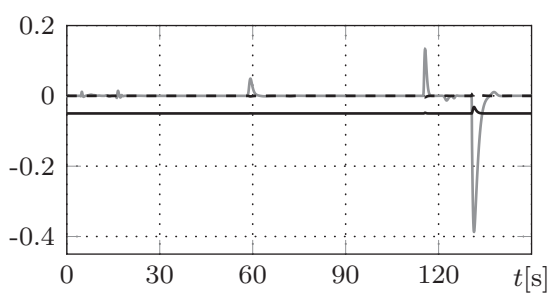

(f) Time evolution of the TF: $f_{x}$ (black line), $f_{y}$ (dashed black line), $f_{\theta}$ (gray line)

Fig. 18. Results of experiment E4 


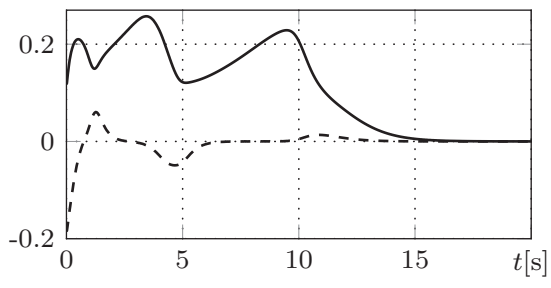

(a) Navigation inputs $v_{x}$ (solid) and $v_{y}$ (dashed) in $[\mathrm{m} / \mathrm{s}]$

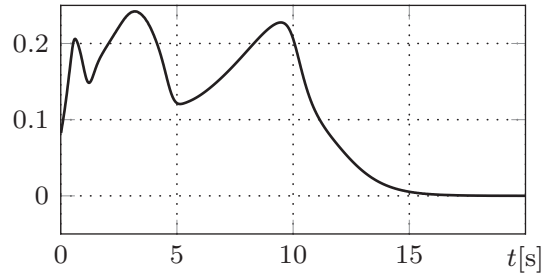

(d) Linear velocity $u_{1}$ of the unicycle in $[\mathrm{m} / \mathrm{s}]$

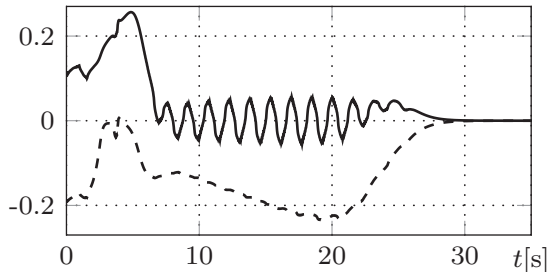

(a) Navigation inputs $v_{x}$ (solid) and $v_{y}$ (dashed) in $[\mathrm{m} / \mathrm{s}]$

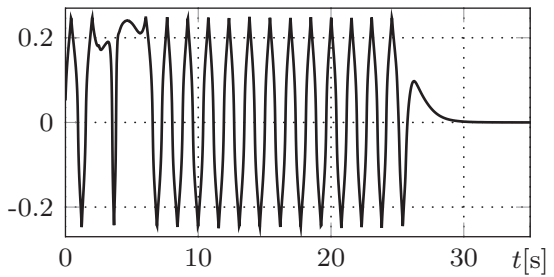

(d) Linear velocity $u_{1}$ of the unicycle in $[\mathrm{m} / \mathrm{s}]$

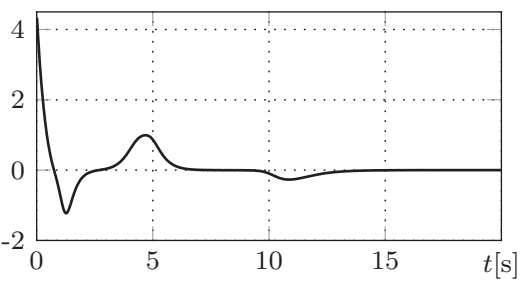

(b) Auxiliary angular input $v_{\theta}[\mathrm{rad} / \mathrm{s}]$

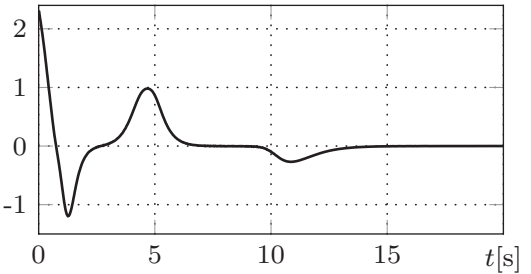

(e) Angular velocity $u_{2}$ of the unicycle in $[\mathrm{rad} / \mathrm{s}]$

Fig. 19. Results of experiment E5

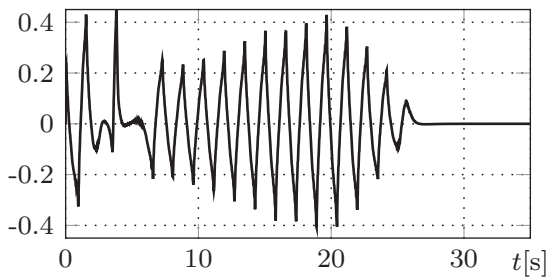

(b) Auxiliary angular input $v_{\theta}[\mathrm{rad} / \mathrm{s}]$

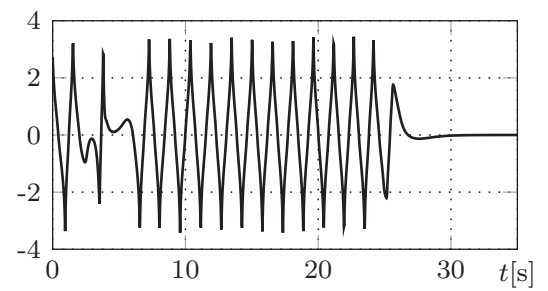

(e) Angular velocity $u_{2}$ of the unicycle in $[\mathrm{rad} / \mathrm{s}]$

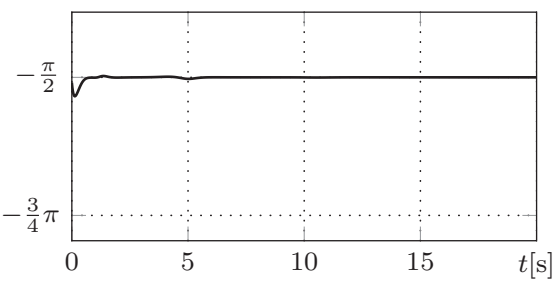

(c) Time evolution of auxiliary variable $\alpha$ in $[\mathrm{rad} / \mathrm{s}]$

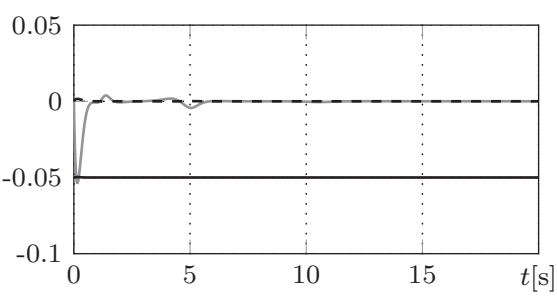

(f) Time evolution of the TF: $f_{x}$ (black line), $f_{y}$ (dashed black line), $f_{\theta}$ (gray line)

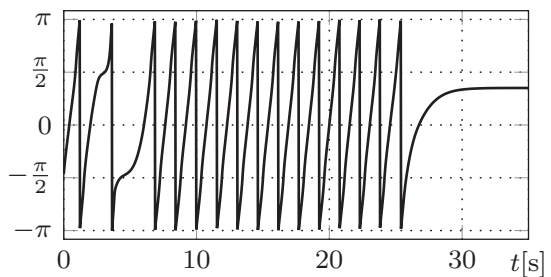

(c) Time evolution of auxiliary variable $\alpha$ in $[\mathrm{rad} / \mathrm{s}]$

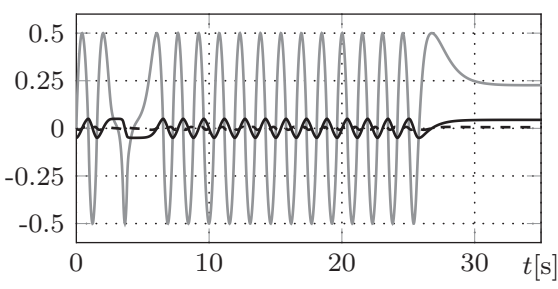

(f) Time evolution of the TF: $f_{x}$ (black line), $f_{y}$ (dashed black line), $f_{\theta}$ (gray line)

Fig. 20. Results of experiment E6

repulsive potential vanishes parameters $\varepsilon_{1}$ increases and the approximation accuracy is relaxed - for example compare the initial trajectory in experiments E2 and E3. It can be confirmed from Fig. $17 \mathrm{f}$ that coordinates $f_{x}$ and $f_{y}$ are scaled during the navigation task. As a result frequency of control input can be attenuated when the robot moves away from obstacles - cf. Figs. 16d, e and 17d, e. It makes it possible to improve performance of the controller and to decrease the oscillatory response if it is not required to accomplish the task.

Experiment E4 illustrates properties of the controller in the trajectory tracking case. The environment structure corresponds to the one used in experiments E1-E3. Similarly as in E1, the heading controller is used and the upper bound of the wheel velocities are limited to $10 \mathrm{rad} / \mathrm{s}$. The goal is assumed to move around a circle with radius $1.5 \mathrm{~m}$ and with linear velocity $0.075 \mathrm{~m} / \mathrm{s}-$ see Fig. 14d. The reference trajectory cyclically collides with three obstacles. From experimental results it follows that the position variables of the virtual robot converge asymptotically to the goal when it is placed outside any obstacle and its corresponding interactive zone. The robot motion does not exhibit oscillatory behaviour - infeasible motion is reduced and the augmented dynamics does not produce oscillation - see Figs. 18c and $\mathrm{f}$. 
In the next experiment, E5, two obstacles with overlapping external zones are considered $-\mathrm{cf}$. Fig. 14e. The position of the stationary goal is defined by $p_{d}=\left[\begin{array}{ll}1 & 1.5\end{array}\right]^{\top}$. Other conditions and parameters correspond to the ones used in experiment E1. This scenario can be interpreted as a motion task through a corridor defined between walls. Since in the considered case the obstacles are not completely isolated the lack of local minima is not guaranteed. In spite of it any local minimum is not met and the robot is attracted to the goal properly in about $17 \mathrm{~s}$. The robot motion is smooth and no rapid changes in the linear velocity are observed - cf. Fig. 19d. As a result of the interaction between the repulsive and attractive fields in the given scenario the robot path is placed in the middle of the feasible passage.

Experiment E6 is a variation of experiment E5. Namely, it is assumed that the robot heading should not be changed significantly during motion execution. To be more precise, it is assumed that orientation of the virtual robot satisfies $z_{\theta}=0$. Comparing Figs. $14 \mathrm{e}$ and $14 \mathrm{f}$ it can be stated that path $\left(z_{x}, z\right.$ $y$ ) is almost the same while in Experiment E5 the robot motion is executed using the zig-zac pattern. Clearly, this kind of motion is less effective and for the same velocity bounds the task is performed in about twice longer - cf. time plots in Figs. 19 and 20.

\section{Conclusions}

The paper studies the application of the controller using transverse functions in order to overcome limitations imposed by nonholonomic constraints. We propose the non classic control structure which can be efficiently used for a differentially driven robot to solve a navigation task. It is important to emphasize that this is a relatively new idea which can be used to execute also non-standard motion tasks where independent orientation control is required. Additionally, it turns out that a possible relaxation of required precision of heading control allows increasing robustness of the closed-loop control system to measurement uncertainties. For example, it is observed that sensitivity of the controller when the robot moves with low velocity is not significant. It confirms that the controller ensures a suitable level of robustness near the equilibrium point.

The discussed stability analysis of the motion controller offers a possibility of finding some tuning rules. In particular one can predict oscillatory response and estimate frequency of a cycle when an infeasible direction is approximated. Additionally, it is possible to avoid zig-zag motion patterns by a proper selection of parameters of the transverse function. These aspects are critical in practice, especially when the robot dynamics including restrictions imposed on acceleration bounds must be taken into account.

The proposed control idea based on approximated decoupling can be adapted for other kinematics including car-like vehicle and vehicle with trailers. Moreover, it can be used to specify fundamental motion patterns which can be employed by a planner to find possible trajectories connecting points in a free task space. Additionally, the considered control strategy can be useful for a navigation purpose using potential approach. In particular, we believe that it could be efficiently used to overcome local minimum of potential functions, similarly as it can be done for holonomic systems. In this case the possibility of approximation of any direction in the state space becomes an important advantage of this method.

\section{A. Appendix}

A.1. Transversality condition - selection of parameter $\varepsilon_{3}$. Let $\rho:=\varepsilon_{2} \cos \alpha \in\left[-\varepsilon_{2}, \varepsilon_{2}\right]$. To simplify our consideration we assume that $\varepsilon_{2} \leq \pi$, that can be easily motivated from a practical point of view.

Using $\rho$ in Eq. (33) gives:

$$
\gamma(\rho)=\frac{1}{\varepsilon_{2}^{2}}\left(\left(\operatorname{sinc} \rho-4 \varepsilon_{3} \cos \rho\right) \rho^{2}+2 \varepsilon_{3} \varepsilon_{2}^{2} \cos \rho\right) .
$$

Suppose that $\varepsilon_{3}=\varepsilon_{3}^{*}(\rho)$ such that $\left.\gamma(\rho)\right|_{\varepsilon_{3}=\varepsilon_{3}^{*}(\rho)}=0$. Consequently, $\varepsilon_{3}^{*}(\rho)$ satisfies

$$
\varepsilon_{3}^{*}(\rho)=\frac{\rho \tan \rho}{2\left(2 \rho^{2}-\varepsilon_{2}^{2}\right)} .
$$

Notice that at $\rho \in\left\{ \pm \frac{\sqrt{2}}{2} \varepsilon_{2}, \pm \frac{\pi}{2}\right\}$ function (93) becomes unbounded. Next, introduce sets

$$
D_{+}:=\left\{\rho \in\left[-\varepsilon_{2}, \varepsilon_{2}\right]: 2 \rho^{2}-\varepsilon_{2}^{2}>0\right\}
$$

and

$$
D_{-}:=\left\{\rho \in\left[-\varepsilon_{2}, \varepsilon_{2}\right]: 2 \rho^{2}-\varepsilon_{2}^{2}<0\right\}
$$

In order to satisfy $\gamma(\rho)>0$ has to satisfy

$$
\sup _{\rho \in D_{+}} \varepsilon_{3}^{*}<\varepsilon_{3}<\inf _{\rho \in D_{-}} \varepsilon_{3}^{*} .
$$

To analyse function $\varepsilon_{3}^{*}(\rho)$ one computes

$$
\begin{aligned}
\frac{d \varepsilon_{3}^{*}(\rho)}{d \rho} & =-\frac{\rho\left(2 \rho^{2}(\operatorname{sinc}(2 \rho)-1)+\varepsilon_{2}^{2}(1+\operatorname{sinc}(2 \rho))\right)}{2\left(\varepsilon_{2}^{2}-2 \rho^{2}\right)^{2} \cos ^{2} \rho} \\
& =\frac{\rho(1+\operatorname{sinc}(2 \rho))\left(2 \rho^{2} \frac{1-\operatorname{sinc}(2 \rho)}{1+\operatorname{sinc}(2 \rho)}-\varepsilon_{2}^{2}\right)}{2\left(\varepsilon_{2}^{2}-2 \rho^{2}\right)^{2} \cos ^{2} \rho}
\end{aligned}
$$

Define $\gamma_{1}(\rho):=2 \rho^{2} \frac{1-\operatorname{sinc}(2 \rho)}{1+\operatorname{sinc}(2 \rho)}$ and investigate roots of the following equation:

$$
\gamma_{1}(\rho)-\varepsilon_{2}^{2}=0
$$

for $\rho \in[-\pi, \pi]$. Taking into account that function $\gamma_{1}(\rho)$ is even, we compute solution to Eq. (98) for positive $\rho$. Recalling 
that $|\rho| \leq \varepsilon_{2}$ from Fig. 21 it can be found that for $\varepsilon_{2} \in\left(0, \bar{\rho}_{1}\right)$, where $\bar{\rho}_{1} \approx 1.1394$ there is no solution to (98). Then, noticing that $\forall \rho \in \mathbb{R}, \operatorname{sinc}(2 \rho)+1<0$ it can be concluded that $\forall \rho \in\left[-\varepsilon_{2}, \varepsilon_{2}\right], \gamma_{1}(\rho)-\varepsilon_{2}^{2}<0$ and there is only one root, $\rho=0$, of equation $\frac{d \varepsilon_{3}^{*}(\rho)}{d \rho}=0$. Consequently, at $\rho=0$ function (93) has local maximum $\varepsilon_{3}^{*}(0)=0$. Since there are no other local extrema in domains $D_{-}$and $D_{+}$one can consider value of (93) at boundary $|\rho|=\varepsilon_{2}-$ cf. Fig. 22 . To be more specific we have: $m_{\varepsilon_{3}}:=\varepsilon_{3}^{*}\left( \pm \varepsilon_{2}\right)=\frac{\tan \varepsilon_{3}}{2 \varepsilon_{3}}$.

Now assume that $\varepsilon_{2} \in\left[\bar{\rho}_{1}, \pi\right]$. In such a case there are real solutions to (98) denoted by $\pm \rho_{m}$. At these points, assuming that $\varepsilon_{2} \neq \bar{\rho}_{2}=\frac{\sqrt{2}}{2} \pi$ (cf. Fig. 21), function (93) has local maxima $m_{\varepsilon_{3}^{*}}:=\varepsilon_{3}^{*}\left( \pm \rho_{m}\right)-$ see Figs. 23 and 24. Simultaneously from Fig. 21 it follows that for $\varepsilon_{2}=\bar{\rho}_{2} \rho_{m}=\frac{\pi}{2}$ and $\varepsilon_{3}^{*}\left( \pm \rho_{m}\right)$ is unbounded. In this particular case a local minimum is not met.

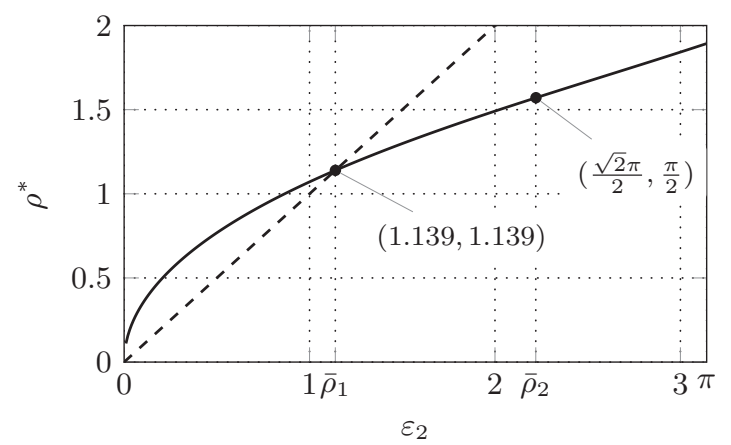

Fig. 21. Root of function $\gamma_{1}(\rho)+\varepsilon_{2}^{2}$

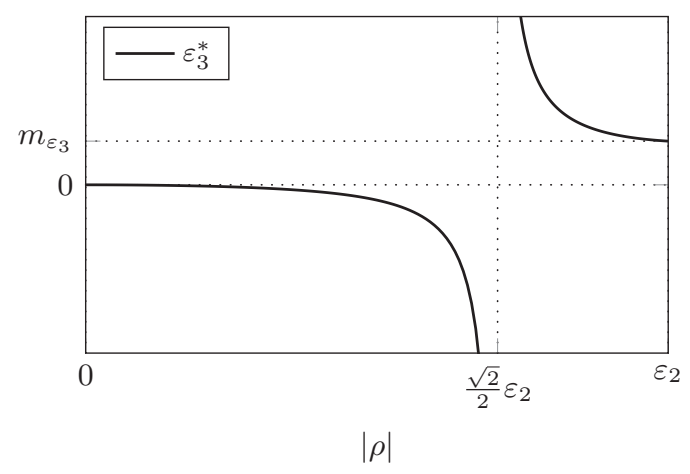

Fig. 22. Plot of function $\varepsilon_{3}^{*}$ for $\varepsilon_{2} \in\left(0, \bar{\rho}_{1}\right)$

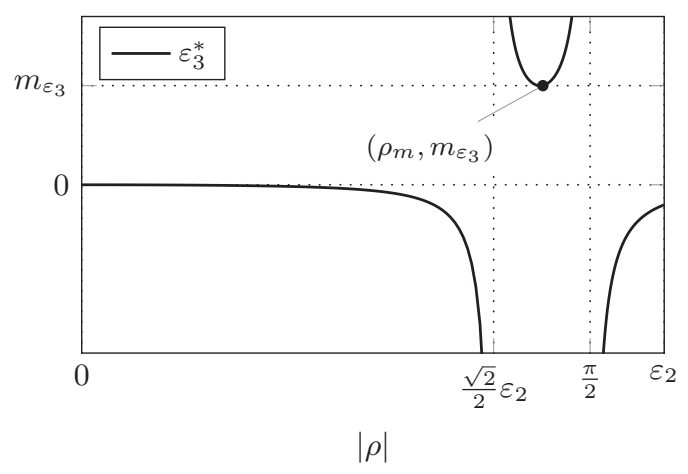

Fig. 23. Plot of function $\varepsilon_{3}^{*}$ for $\varepsilon_{2} \in\left[\bar{\rho}_{1}, \bar{\rho}_{2}\right]$

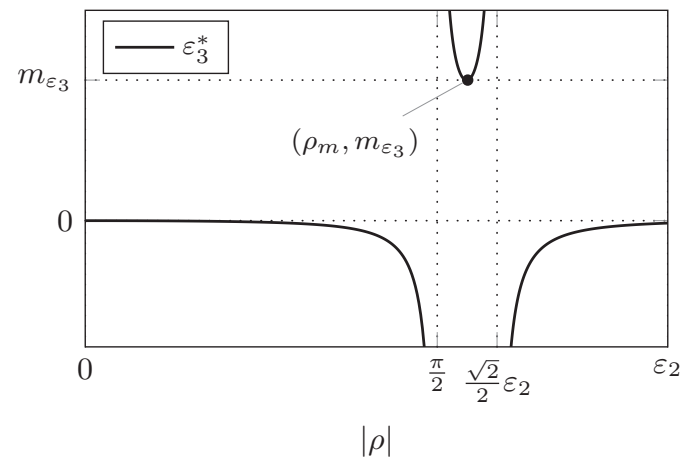

Fig. 24. Plot of function $\varepsilon_{3}^{*}$ for $\varepsilon_{2} \in\left(\bar{\rho}_{2}, \pi\right]$

Recalling inequality (96) one can formulate the following constraint: $\varepsilon_{3}<m_{\varepsilon_{3}^{*}}$, where

$$
m_{\varepsilon_{3}}= \begin{cases}\frac{1}{2} \operatorname{tanc} \varepsilon_{2} & \text { if } \varepsilon_{2} \in\left(0, \bar{\rho}_{1}\right) \\ \varepsilon_{3}^{*}\left( \pm \rho_{M}\right) & \text { if } \varepsilon_{2} \in\left(\bar{\rho}_{1}, \pi\right)\end{cases}
$$

with $\operatorname{tanc} \sigma:=\frac{\tan \sigma}{\sigma}$ for $\sigma \in \mathbb{R}$.

A.2. Analysing augmented dynamics - description of function $\boldsymbol{\kappa}_{1}$. Defining $\rho:=\varepsilon_{2} \cos \alpha \in\left[-\varepsilon_{2}, \varepsilon_{2}\right]$ and using this term in (46) yields

$$
\kappa_{1}(\rho):=\cos (\rho)+2 \varepsilon_{3} \rho \sin (\rho)
$$

Next, assume that $\kappa_{1}\left(\rho^{*}\right)=0$ and compute

$$
\varepsilon_{3}(\rho)=-\frac{1}{2 \rho \tan \rho} .
$$

Obviously $\varepsilon_{3}(\rho)$ is unbounded when $\rho \rightarrow 0$. Computing derivative of $\varepsilon_{3}(\rho)$ one has

$$
\varepsilon_{3}^{\prime}(\rho)=\frac{\frac{1}{2} \sin 2 \rho+\rho}{2(\rho \sin \rho)^{2}}=\frac{\operatorname{sinc}(2 \rho)+\frac{1}{2}}{2 \rho \sin ^{2} \rho} .
$$

Noticing that there is no solution in real domain for $(2 \rho)+\frac{1}{2}=0$ we conclude that function $\varepsilon_{3}(\rho)$ does not have extrema. Hence minimum values in the range $\rho \in\left[-\varepsilon_{2}, \varepsilon_{2}\right]$ can be found at the boundaries, namely

$$
\inf _{\rho \in\left[-\varepsilon_{2}, \varepsilon_{2}\right]} \varepsilon_{3}=\varepsilon_{3}\left( \pm \varepsilon_{2}\right)=-\frac{1}{2 \varepsilon_{2} \tan \varepsilon_{2}} .
$$

\section{REFERENCES}

[1] R.W. Brockett, "Asymptotic stability and feedback stabilization”, in Differential geometric control theory, 181-191, Birkhäuser, Boston, 1983.

[2] C. Canudas de Wit, H. Khennouf, C. Samson, and O.J. Sørdalen, "Nonlinear control design for mobile robots", in Recent trends in mobile robots, 121-156, World Scientific, Singapore, 1993. 
[3] R. Fierro and F.L. Lewis, "Control of a nonholonomic mobile robot: backstepping kinematics into dynamics", Proc. 34th IEEE Conf. on Decision and Control, 3805-3810 (1995).

[4] G. Oriolo, A. De Luca, and M. Venditteli, "WMR control via dynamic feedback linearization: design, implementation and experimental validation", IEEE Trans. Control Syst. Technol. 10 (6), 835-852 (2002).

[5] M. Michałek and K. Kozłowski, "Vector-Field-Orientation feedback control method for a differentially driven vehicle", IEEE Trans. Control Syst. Technol. 18 (1), 45-65 (2010).

[6] C. Samson, "Velocity and torque feedback control of a nonholonomic cart", in Advanced robot control, 125-151, Birkhauser, Boston, MA, 1991.

[7] J.B. Pomet, "Explicit design of time varying stabilization control laws for a class of controllable systems without drifts", Syst. Control Lett. 18 (2), 147-158 (1992).

[8] C. Canudas de Wit and O.J. Sørdalen, "Exponential stabilization of mobile robots with nonholonomic constraints", IEEE Trans. Autom. Control 37 (11), 1791-1797 (1992).

[9] A. Astolfi, "Asymptotic stabilization of nonholonomic systems with discontinuous control", $\mathrm{PhD}$ thesis, Swiss Federal Intitute of Technology, Zurich, 1996.

[10] D.A. Lizárraga, "Obstructions to the existence of universal stabilizers for smooth control systems", Math. Control Signal. 16 (4), 255-277 (2004).

[11] P. Morin and C. Samson, "A characterization of the Lie Algebra Rank Condition by transverse periodic functions", SIAM J. Control Optim. 40 (4), 1227-1249 (2001).

[12] P. Morin and C. Samson, "Control of nonholonomic mobile robots based on the transverse function approach", IEEE Trans. Robot. 25 (5), 1058 -1073 (2009).

[13] P. Morin and C. Samson, "Transverse function control of a class of non-invariant driftless systems. application to vehicles with trailers", Proc. 47th IEEE Conf. on Decision and Control, 4312-4319 (2008)

[14] D. Pazderski, K. Kozłowski, and D.K. Waśkowicz, "Control of a unicycle-like robot with trailers using transverse function approach", Bull. Pol. Ac.: Tech. 60 (3), 537-546 (2012).

[15] P. Morin and C. Samson, "Stabilization of trajectories for systems on Lie groups. Application to the rolling sphere", Proc. 17th IFAC World Congress, 508-513 (2008).

[16] M. Ishikawa, P. Morin, and C. Samson, "Tracking control of the trident snake robot with the transverse function approach", Proc. 48th IEEE Conf. on Decision and Control and 28th Chinese Control Conf., 4137-4143 (2009).

[17] W. Magiera, "Nonsymetric trident snake: the transverse function approach", Robotics Advances 1, 451-460 (2012), (in Polish).

[18] D. Pazderski and K. Kozłowski, "Control of planar robot in the flight phase using transverse function approach", Bull. Pol. Ac.: Tech. 63 (3), 759-770 (2015).

[19] B. Krysiak and K. Kozłowski, "Kinematic singularities avoidance for the planar three-link nonholonomic manipulator", Proc. 10th Int. Workshop on Robot Motion and Control, 252- 256 (2015).

[20] J. Jakubiak, W. Magiera, and K. Tchoń, "Control and motion planning of a nonholonomic parallel orienting platform", $A S M E$. J. Mechanisms Robotics 7 (4), (2015), DOI:10.1115/1.4029891.

[21] D.A. Lizárraga, P. Morin, and C. Samson, "Non-robustness of continuous homogeneous stabilizers for affine control systems", Proc. 37th IEEE Conf. on Decision and Control, 855- 860 (1999).
[22] Z.-P. Jiang, "Robust exponential regulation of nonholonomic systems with uncertainties", Automatica 36 (2), 189-209 (2000).

[23] D. Pazderski, B. Krysiak, and K. Kozłowski, "A comparison study of discontinuous control algorithms for three-link nonholonomic manipulator", Lecture Notes in Control and Inform. Sci. Robot Motion Control: Recent Developments 396, 35-44 (2012).

[24] I. Dulęba, Algorithms of Motion Planning for Nonholonomic Robots, Publishing House of Wrocław University of Technology, Wrocław, 1998, (in Polish).

[25] S. M. LaValle, Planning Algorithms. Cambridge University Press, Cambridge, UK, 2006.

[26] O. Khatib, "Real-time obstacle avoidance for manipulators and mobile robots", Int. J. Robot. Res. 5 (1), 90-98 (1986).

[27] D. Koditschek, "Exact robot navigation by means of potential functions: Some topological considerations", Proc. IEEE Int. Conf. Robot. and Autom., 1-6 (1987).

[28] E. Rimon and D.E. Koditschek, "Exact robot navigation using artificial potential fields", IEEE Trans. Robot. and Autom. 8 (5), 501-518 (1992).

[29] T. Urakubo, K. Okuma, and Y. Tada, "Feedback control of a two wheeled mobile robot with obstacle avoidance using potential functions", Proc. IEEE/RSJ Int. Conf. on Intelligent Robots and Systems, 2428-2433 (2004).

[30] W. Kowalczyk, M. Michałek, and K. Kozłowski, "Trajectory tracking control with obstacle avoidance capability for unicycle-like mobile robot", Bull. Pol. Ac.: Tech. 60 (3), 537-546 (2012).

[31] J. Kim and P. Khosla. "Real-time obstacle avoidance using harmonic potential functions", IEEE Trans. Robot. Autom. 8 (3), 338-349 (1992).

[32] S. Waydo and R. M. Murray, "Vehicle motion planning using stream functions", Proc. IEEE Int. Conf. Robot. and Autom., 2484-2491 (2003).

[33] R. Soukieh, I. Shames, and B. Fidan. "Obstacle avoidance of non-holonomic unicycle robots based on fluid mechanical mod18 Bull. Pol. Ac.: Tech. XX(Y) 2016 Application of transverse functions eling", Proc. European Control Conf., 3269-3274 (2009).

[34] P. Szulczyński, D. Pazderski, and K. Kozłowski, "Obstacle avoidance and trajectory tracking using fluid-based approach in 2D space", Lecture Notes in Control and Inform. Sci. Robot Motion and Control 2011 422, 293-304 (2012).

[35] S. Akishita, S. Kawamura, and K Hayashi. "Laplace potential for moving obstacle avoidance and approach of a mobile robot", Proc. Japan-USA Symposium on Flexible Automation/A Pacific Rim Conference, 139-142 (1990).

[36] C.I. Connolly, J.B. Burns, and R. Weiss, "Path planning using Laplace's equation", Proc. IEEE Int. Conf. on Robot. Autom., 2102-2106 (1990).

[37] D. Panagou, H.G. Tanner, and K.J. Kyriakopoulos, "Dipolelike fields for stabilization of systems with Pfaffian constraints", Proc. IEEE Int. Conf. Robot. Autom., 4499-4504 (2010).

[38] S. Mastellone, D.M. Stipanović, C.R. Graunke, K.A. Intlekofer, and M.W. Spong, "Formation control and collision avoidance for multi-agent non-holonomic systems: Theory and experiments", Int. J. Robot. Res. 27 (1), 107-126 (2008). 
[39] D. Panagou. "Dipole-like fields for stabilization of systems with Pfaffian constraints", Proc. IEEE Int. Conf. Robot. and Autom., 2513 - 2518 (2014).

[40] E.J. Rodríguez-Seda, C. Tang, M.W. Spong, and D.M. Stipanović, "Trajectory tracking with collision avoidance for nonholonomic vehicles with acceleration constraints and limited sensing", Int. J. Robot. Res. 33 (12), 1569-1592 (2014).

[41] G. Artus, P. Morin, and C. Samson, "Tracking of an omnidirectional target with a unicycle-like robot: control design and experimental results", Research Report INRIA 4849, INRIA, 2003.

[42] D. Pazderski, D.K. Waśkowicz, and K. Kozłowski, "Motion control of vehicles with trailers using transverse function approach", J. Intell. Robotic Syst. 77 (3-4), 457-479 (2015).
[43] P. Morin and C. Samson, "Practical stabilization of driftless systems on Lie groups: the transverse function approach", IEEE Trans. Autom. Control 48 (9), 1496-1508 (2003).

[44] H. Hermes. "Nilpotent and high-order approximations of vector field systems", SIAM Rev. 33 (2), 238-264 (1991).

[45] R. Volpe and P. Khosla, "Artificial potentials with elliptical isopotential contours for obstacle avoidance", Proc. 26th IEEE Conf. Decision and Control, 180-185 (1987).

[46] K. Kozłowski,W. Kowalczyk, B. Krysiak, M. Kiełczewski, and T. Jedwabny, "Modular architecture of the multi-robot system for teleoperation and formation control purposes", In Proc. 9th Int. Workshop Robot Motion and Control, 19-24 (2013).

[47] A.H. Barr, "Superquadrics and angle-preserving transformations", IEEE Comput. Graph. Appl. 1 (1), 11-23 (1981). 